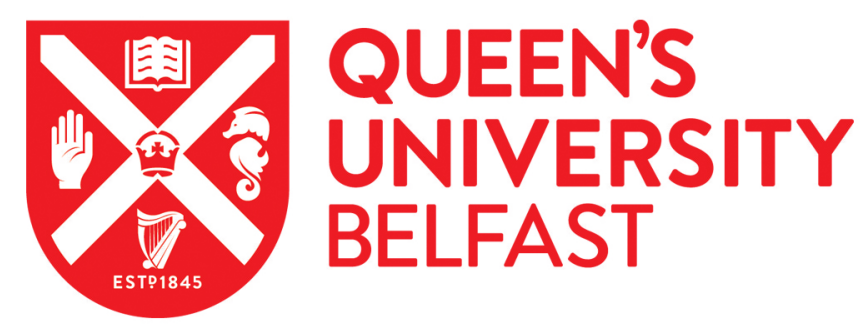

\title{
Design and Analysis of Full-Duplex Massive Antenna Array Systems Based on Wireless Power Transfer
}

Mohammadi, M., Chalise, B. K., Suraweera, H. A., Ngo, H. Q., \& Ding, Z. (2021). Design and Analysis of FullDuplex Massive Antenna Array Systems Based on Wireless Power Transfer. IEEE Transactions on Communications, 69(2), 1302-1316. https://doi.org/10.1109/TCOMM.2020.3035401, https://doi.org/10.1109/TCOMM.2020.3035401

Published in:

IEEE Transactions on Communications

Document Version:

Peer reviewed version

Queen's University Belfast - Research Portal:

Link to publication record in Queen's University Belfast Research Portal

Publisher rights

Copyright 2020 IEEE. This work is made available online in accordance with the publisher's policies. Please refer to any applicable terms of use of the publisher.

\section{General rights}

Copyright for the publications made accessible via the Queen's University Belfast Research Portal is retained by the author(s) and / or other copyright owners and it is a condition of accessing these publications that users recognise and abide by the legal requirements associated with these rights.

Take down policy

The Research Portal is Queen's institutional repository that provides access to Queen's research output. Every effort has been made to ensure that content in the Research Portal does not infringe any person's rights, or applicable UK laws. If you discover content in the Research Portal that you believe breaches copyright or violates any law, please contact openaccess@qub.ac.uk. 


\title{
Design and Analysis of Full-Duplex Massive Antenna Array Systems Based on Wireless Power Transfer
}

\author{
Mohammadali Mohammadi, Member, IEEE, Batu K. Chalise, Senior Member, IEEE, \\ Himal A. Suraweera, Senior Member, IEEE, Hien Quoc Ngo, Senior Member, IEEE, \\ and Zhiguo Ding, Fellow, IEEE
}

\begin{abstract}
In this paper, we consider a wireless communication system, where a full-duplex hybrid access point (HAP) transmits to a set of cellular users (CUs) in the downlink channel, while receiving data from a set of energy-constrained communication devices like user equipments (UEs) in the uplink channel. The HAP has a massive antenna array, while all CUs and UEs nodes are equipped with single antenna each. Time switching protocol is adopted, where channel estimation, wireless power transfer, and information transfer between UEs, CUs and full-duplex HAP are performed in two phases. By adopting maximum ratio combining/maximum ratio transmission (MRC/MRT) and zero-forcing ( $\mathrm{ZF}$ ) processing at the HAP, the uplink and downlink achievable rate expressions in the large-antenna limit and approximate results that hold for any finite number of antennas are derived. Moreover, the optimum energy beamformer and time-split parameter at the HAP are found to maximize the downlink sum-rate under a constraint on uplink sum-rate. Our findings reveal that our proposed energy beamforming with $\mathrm{ZF}$ and MRC/MRT processing for information transfer achieves up to $47 \%$ and $14 \%$ average sum rate gains as compared with the suboptimum energy beamformer, respectively.
\end{abstract}

Index Terms-Full-duplex (FD), beamforming, massive multiple-input multiple-output, wireless power transfer.

\section{INTRODUCTION}

New generation wireless standards are poised to deliver higher data rates and support massive number of connections using innovative technologies. For example, massive multiple-input multiple-output (MIMO) has become one of

Manuscript received July 15, 2020; revised Oct. 01, 2020 and Oct. 19, 2020; accepted Oct. 27, 2020. Part of this work has been presented at the IEEE International Conference on Communications (ICC 2017), Paris, France, June. 2017 [1]. The work of M. Mohammadi was supported in part by the Research Deputy of Shahrekord University under grant number 97GRN1M1087 and 99GRN1M1087. The associate editor coordinating the review of this paper and approving it for publication was Prof. Ana Garcia Armada. (Corresponding author: Mohammadali Mohammadi.)

M. Mohammadi is with the Faculty of Engineering, Shahrekord University, Shahrekord 115, Iran (email: m.a.mohammadi@sku.ac.ir).

B. K. Chalise is with Department of Electrical and Computer Engineering, New York Institute of Technology, Northern Boulevard, Old Westbury, NY 11568 (email: batu.k.chalise@ieee.org).

H. A. Suraweera is with the Department of Electrical and Electronic Engineering, University of Peradeniya, Peradeniya 20400, Sri Lanka (email: himal@ee.pdn.ac.lk).

H. Q. Ngo is with the Institute of Electronics, Communications \& Information Technology (ECIT), Queen's University Belfast, Belfast BT3 9DT, U.K. (email: hien.ngo@qub.ac.uk).

Z. Ding is with School of Electrical and Electronic Engineering, The University of Manchester, Manchester, M13 9PL, U.K (e-mail: zhiguo.ding@manchester.ac.uk). the core components to be incorporated in the fifth generation $(5 \mathrm{G})$ communication systems. According to the massive MIMO paradigm, base stations (BSs) are equipped with large antenna arrays to serve a large number of communication devices with high quality of services [2]. These devices are not only restricted to smart mobile phones as they also embrace sensors, electronic appliances and other machinetype user equipment (UE) with sensing, processing, and communication capabilities. Such devices are often deployed in distributed environments or even hard-to-reach places, thus replacing their batteries incurs substantial cost and effort overhead. To deal with this challenge, devices have to be self-sustained in terms of their energy requirements, for example, they could use energy harvesting techniques [3].

Meanwhile, in-band full-duplex (FD) communication has been promoted as an attractive technology to satisfy the exponential growth of spectral efficiency requirements of future wireless networks [4]. FD transmissions under high signal-to-noise ratio (SNR) can potentially double the wireless spectral efficiency of conventional half-duplex (HD) systems by enabling simultaneous transmission and reception over the same frequency band. However, the self-interference (SI) from transmit antennas to receive antennas may severely degrade the performance of FD transceivers [5], [6]. Moreover, FD operation in cellular systems causes additional interferences as the simultaneous active links are increased compared to the conventional HD cellular systems. More precisely, the downlink rates will be degraded by new interference from uplink users and uplink rates will be affected by the SI and new interference from neighbouring FD BSs [7]. Today, due to advanced hardware processing techniques, SI cancellation methods including wireless-propagation domain techniques [8], complemented by analog-circuit-domain and digital-domain techniques, have made FD operation feasible [4]. Moreover, spatial domain SI cancellation solutions through the use of beamforming techniques have been proposed and implemented in wireless FD systems [5], [9]. Intra-cell and inter-cell interference management in FD enabled cellular networks has been investigated in [10][13]. Specifically, [10] investigated the potential use of massive antenna arrays at the BSs with FD operation. The authors in [11] proposed the use of directional antennas to mitigate the interference in FD cellular networks. In [12], with the aim of satisfying fairness requirements, transmit and receive beamforming design in multi-cell FD systems was 
studied. In [13], the authors have developed an interference management mechanism via uplink power optimization in FD cellular networks.

Massive antenna arrays can help to form sharp energy beams towards users/devices to improve the energy efficiency of wireless networks and thus their application in wireless information and energy transfer systems has been widely investigated [14]-[20]. Specifically, the authors of [14] jointly optimized the energy allocation weights in downlink energy transfer phase and the fraction of energy used for channel estimation such that a minimum rate among uplink users is maximized. In [15], the minimum amount of harvested energy was maximized among the energy users, while the rate requirements of information users are guaranteed. In [16], feasibility of wireless power transfer (WPT) in massive MIMO-enabled sensor networks has been studied and the outage probability due to energy harvesting was characterized. In [17], the minimum achievable rate among all the users has been maximized, by optimizing the power allocated to the BS and power splitting factor at the users. An efficient power allocation and beamforming design was proposed in [18], that improves the energy harvesting efficiency of energy users, while satisfying the outage probability requirements of information users. In [19], WPT energy efficiency optimization problem was formulated and solved in a multi-user massive MIMO system with regard to beamforming design, antenna selection, power allocation, and time division protocol in presence of imperfect channel estimation. The authors in [20] optimized the system energy efficiency by joint power allocation design at the BS and the power splitting or time switching parameters at the receivers.

Common to all of the above works [14]-[20] is the HD operation assumption at the massive MIMO BS. Recently, the potential of FD radio in massive MIMO wireless powered communication networks has been explored in [21], [22]. In particular, in order to maximize the sum rate and energy efficiency of the FD system, path-following algorithms were developed in [21] through jointly designing the energy harvesting time and beamforming. The authors in [22] proposed a hybrid time switching (TS) and power splitting (PS) simultaneous wireless information and power transfer (SWIPT) protocol design in a FD massive MIMO system. Specifically, a beam-domain hybrid SWIPT protocol was designed in [22], where downlink users and uplink sensors are intelligently scheduled according to their beamdomain channel distributions to enhance overall transmission spectral efficiency. By optimizing transmit powers at the BS during the two phases and the TS ratio, the system achievable sum-rate is maximized.

In this paper, unlike [21], [22], we study the impact of imperfect channel state information (CSI) on the performance of a FD massive MIMO system consisting an FD HAP, energy-constrained sensors/user equipments (UEs), and a set of cellular users (CUs). The communication is carried out in two transmission phases in the same time slot and over the same frequency band. In the first phase, HAP transfers energy to UEs and at the same time receives pilot symbols transmitted by CUs. In the second phase, the HAP estimates the uplink channels and uses the channel estimates to design the transmit beamformer for downlink transmission to all CUs, while receiving information from UEs. We assume a massive antenna array at the HAP as a practical assumption [16]. We obtain the uplink and downlink achievable rates. Moreover, downlink-uplink sum-rate region is characterized by optimizing the energy beamformer and time-split parameter at the HAP. Specifically, the downlink sum-rate is maximized by ensuring that the uplink sum-rate is above a certain threshold.

The contributions of this paper are three-fold:

- We derive new expressions in closed-form for the achievable uplink and downlink rates which are applicable for large-antenna regime at the HAP that employs maximum ratio combining (MRC), maximum ratio transmission (MRT) processing, and zero-forcing (ZF). In order to provide important insights into the system performance and highlight the system behavior, approximate results that hold for any finite number of antennas for imperfect and perfect CSI cases are provided.

- Our results show that in the limit of infinitely many receive antennas at the $\mathrm{HAP}, N \rightarrow \infty$, and energy harvesting, the HAP transmit power can be scaled down proportionally to $1 / N^{2}$. Moreover, for large transmit antenna arrays, $M, \mathrm{ZF}$ processing provides a constant gain over the MRC/MRT processing which depends on the channel estimation errors at the BS.

- In order to maintain a tradeoff between energy transfer to UEs and the interference they generate to downlink transmission from HAP, we formulate joint energy beamforming design and energy harvesting time and solve it using an efficient method. Energy beamforming design is solved based on successive convex approximation (SCA) and semidefinite relaxation (SDR) for the beamformer and line search is used to find the time-split parameter.

In our conference paper [1], we derived the uplink and downlink achievable rates for MRC/MRT processing under a short-term transmit power constraint. Additionally in this work, we have presented achievable uplink and downlink rates for MRC/MRT processing and $\mathrm{ZF}$ processing under a long-term transmit power constraint. Furthermore, we have generalized our proposed energy beamformer and time-split design for both MRC/MRT and ZF processing under an imperfect CSI assumption.

The rest of the paper is organized as follows. The system model together with the channel estimation and energy harvesting phases are described in Section II. Section III presents the results for the achievable rate of both downlink and uplink transmissions. In Section IV optimum energy beamforming design is studied. Numerical results are presented in Section V, followed by conclusions in Section VI. 


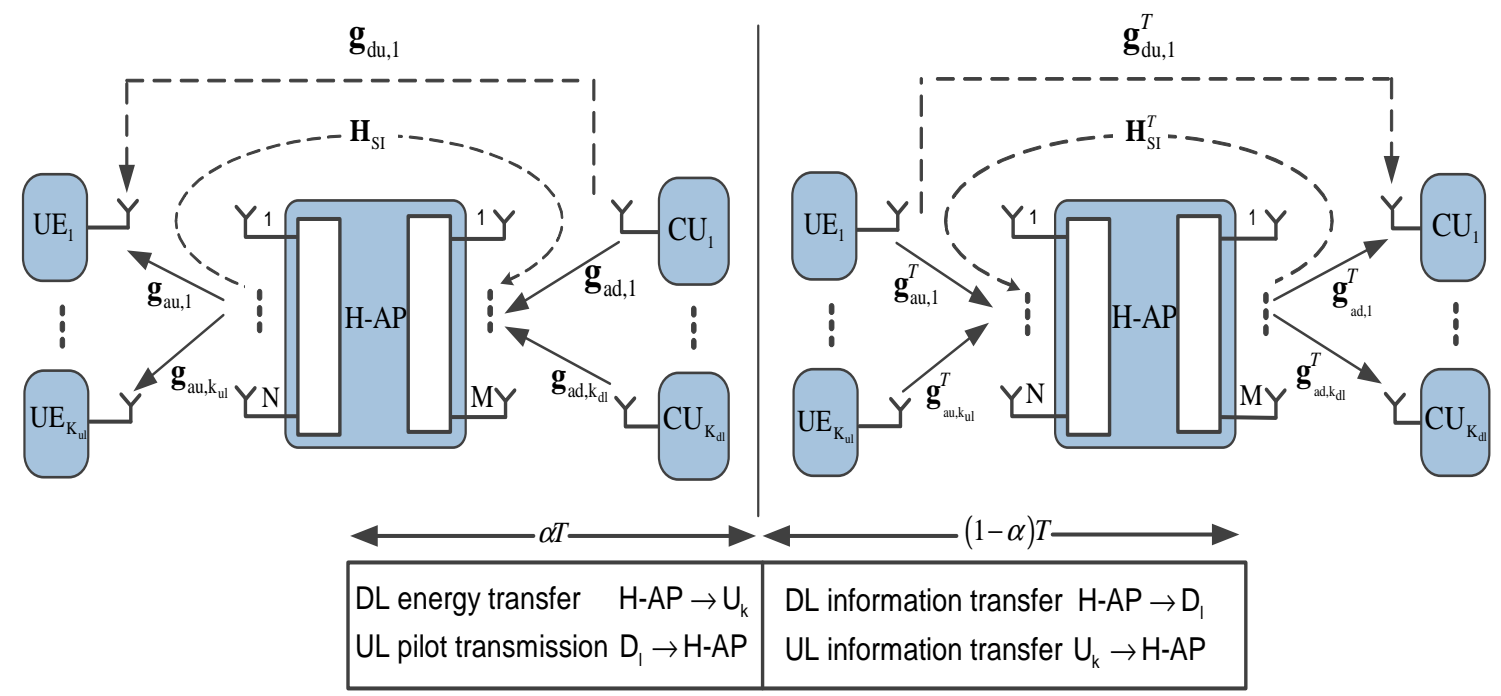

Fig. 1: FD HAP system model and protocol.

Notation: We use bold upper case letters to denote matrices, bold lower case letters to denote vectors, and lower case letters to denote scalers. Moreover, $(\cdot)^{\dagger},(\cdot)^{T}$, and $(\cdot)^{-1}$ represent the conjugate transpose operator, the transpose operator, and the matrix inverse, respectively; Also, $\|\cdot\|$ is the Euclidean norm $\operatorname{tr}(\cdot)$ represents the trace of the matrix, and $\operatorname{vec}(\cdot)$ denotes the vectorization operation of the matrix. In addition, $\otimes$ denotes the matrix Kronecker product and $\mathbf{A}=\operatorname{diag}\left\{\mathbf{A}_{1}, \cdots, \mathbf{A}_{n}\right\}$ stands for a block diagonal matrix. Finally, the statistical expectation and variance operators are represented by $\mathrm{E}\{\cdot\}$ and $\operatorname{VAR}(\cdot)$, respectively. $\mathcal{C N}\left(0, \sigma^{2}\right)$ denotes a circular symmetric complex Gaussian random variable (RV) with zero mean and variance $\sigma^{2}$.

\section{System Model}

\section{A. Network Topology}

We consider a wireless powered communication network, as shown in Fig. 1, where one FD HAP simultaneously serves $K_{\mathrm{dl}}$ downlink users (denoted as CUs) and $K_{\mathrm{ul}}$ communication devices (denoted as UEs). The HAP is equipped with $N$ receive antennas and $M$ transmit antennas. The CUs and UEs have single antenna while the each UE employs a rectenna for energy harvesting. The harvested energy at each UE is used to power the subsequent uplink data transmission. A well recognized $5 \mathrm{G}$ use case is the coexistence of machine type devices and cellular users and how to power up such devices for efficient data transfer is an important issue. To this end, the considered system model has been actively considered in the present literature such as [21]-[24].

\section{B. Signal Transmission Model}

We assume that transmissions are organized in frames over quasi-static flat fading channels on the same frequency band. The length of one frame is fixed to $T$ seconds. As shown in Fig. 1, each frame is divided into two phases. During the first fraction $0 \leq \alpha \leq 1$ of time frame, CUs transmit orthogonal pilots and HAP estimates the uplink channels, while the HAP simultaneously delivers energy and UEs harvest energy from the received signal. By exploiting the channel reciprocity, the HAP obtains the downlink CSI and then forms the beamformers for information transmission to the CUs during the remaining fraction $(1-\alpha)$. Moreover, by using the harvested energy, UEs send their data during the interval $(1-\alpha) T$.

1) Channel Estimation and Energy harvesting: Let $\tau=$ $\alpha T$ be the length of training part for each frame in symbols. In the training phase, all CUs simultaneously transmit pilot sequences, while HAP delivers energy to the UEs via beamforming. The pilot sequences transmitted by the $K_{\mathrm{dl}}$ CUs is represented by $\boldsymbol{\Phi}_{\mathrm{d}} \in \mathbb{C}^{K_{\mathrm{dl}} \times \tau}\left(\tau \geq K_{\mathrm{dl}}\right)$ which satisfies $\boldsymbol{\Phi}_{\mathrm{d}} \boldsymbol{\Phi}_{\mathrm{d}}^{\dagger}=\mathbf{I}_{K_{\mathrm{dl}}}$. The received pilot matrix at the HAP can be written as

$$
\mathbf{Y}_{\mathrm{a}}=\sqrt{\tau p_{p}} \mathbf{G}_{\mathrm{da}} \mathbf{\Phi}_{\mathrm{d}}+\sqrt{\tau p_{\mathrm{a}}} \sum_{k=1}^{K_{\mathrm{ul}}} \mathbf{H}_{\mathrm{SI}} \mathbf{w}_{\mathrm{E}, k} \mathbf{s}_{\mathrm{E}}+\mathbf{N}_{\mathrm{ad}}
$$

where $p_{p}$ pilot symbol's power; $\mathbf{G}_{\mathrm{da}}=\left[\mathbf{g}_{\mathrm{da}, 1}, \cdots, \mathbf{g}_{\mathrm{da}, K_{\mathrm{dl}}}\right] \in$ $\mathbb{C}^{M \times K_{\mathrm{dl}}}$ is the channel matrix between the CUs and the HAP; $\mathbf{H}_{\mathrm{SI}} \in \mathbb{C}^{M \times N}$ denotes the SI channel with independent entries drawn from a $\mathcal{C N}\left(0, \sigma_{\mathrm{SI}}^{2}\right)$ distribution [5], [9]; $p_{\text {a }}$ is the average transmit power of the HAP for energy symbol; $\mathbf{w}_{\mathrm{E}, k} \in \mathbb{C}^{N \times 1}$ denotes the energy beamforming vector for the $k$-th $\mathrm{UE}$ with $\left\|\mathrm{w}_{\mathrm{E}, k}\right\|=1 ; \mathrm{s}_{\mathrm{E}} \in \mathbb{C}^{1 \times \tau}$ denotes the energy sequence transmitted by the HAP for the UEs; and $\mathbf{N}_{\mathrm{ad}}$ is the $M \times \tau$ noise matrix at the HAP. Moreover, $\mathbf{G}_{\mathrm{da}}$ can be expressed as $\mathbf{G}_{\mathrm{da}}=\mathbf{H}_{\mathrm{da}} \mathbf{D}_{\mathrm{da}}^{1 / 2}$, where $\mathbf{H}_{\mathrm{da}} \in \mathbb{C}^{M \times K_{\mathrm{dl}}}$ has i.i.d. $\mathcal{C N}(0,1)$ elements and stands for small-scale fading matrix, while $\mathbf{D}_{\mathrm{da}}$ is the large-scale fading diagonal matrix whose $k$-th diagonal element is denoted by $\beta_{\mathrm{da}, k}$

We can further express (1) as

$$
\mathbf{Y}_{\mathrm{a}}=\overline{\mathbf{H}} \mathbf{X}_{p}+\mathbf{N}_{\mathrm{ad}}
$$


where $\overline{\mathbf{H}}=\left[\mathbf{G}_{\mathrm{da}}, \mathbf{H}_{\mathrm{Sl}}\right]$ is the $M \times\left(K_{\mathrm{dl}}+N\right)$ overall channel matrix, $\mathbf{X}_{p}=\left[\sqrt{\tau p_{p}} \mathbf{\Phi}_{\mathrm{d}} ; \sqrt{\tau p_{\mathrm{a}}} \mathbf{\Phi}_{\mathrm{E}}\right]$ with $\boldsymbol{\Phi}_{\mathrm{E}}=$ $\sum_{k=1}^{K_{\mathrm{ul}}} \mathbf{w}_{\mathrm{E}, k} \mathbf{S}_{\mathrm{E}}$ is the $\left(K_{\mathrm{dl}}+N\right) \times \tau$ signal matrix. Given, $\mathbf{Y}_{\mathrm{a}}$, minimum mean-square-error (MMSE) estimate of $\overline{\mathbf{H}}$ can be written as $\hat{\mathbf{H}}=\left[\hat{\mathbf{G}}_{\mathrm{da}}, \hat{\mathbf{H}}_{\mathrm{SI}}\right]$, where $^{1}$

$$
\begin{aligned}
\mathbf{G}_{\mathrm{da}} & =\hat{\mathbf{G}}_{\mathrm{da}}+\mathcal{E}_{\mathrm{da}}, \\
\mathbf{H}_{\mathrm{SI}} & =\hat{\mathbf{H}}_{\mathrm{SI}}+\mathcal{E}_{\mathrm{SI}},
\end{aligned}
$$

where matrices $\mathcal{E}_{\mathrm{da}}$ and $\mathcal{E}_{\mathrm{SI}}$ denote the i.i.d. Gaussian estimation error. According to property of MMSE channel estimation $\hat{\mathbf{G}}_{\mathrm{da}}, \mathcal{E}_{\mathrm{da}}, \hat{\mathbf{H}}_{\mathrm{SI}}$, and $\mathcal{E}_{\mathrm{SI}}$ are independent [26]. Moreover, the rows of $\hat{\mathbf{G}}_{\mathrm{da}}, \mathcal{E}_{\mathrm{da}}, \hat{\mathbf{H}}_{\mathrm{SI}}$, and $\mathcal{E}_{\mathrm{SI}}$ are mutually independent and distributed as $\mathcal{C N}\left(\mathbf{0}, \boldsymbol{\Omega}_{\mathrm{da}}\right), \mathcal{C N}\left(\mathbf{0}, \mathbf{D}_{\mathrm{da}}-\right.$ $\left.\boldsymbol{\Omega}_{\mathrm{ad}}\right), \mathcal{C N}\left(\mathbf{0}, \boldsymbol{\Omega}_{\mathrm{SI}}\right)$, and $\mathcal{C N}\left(\mathbf{0}, \sigma_{\mathrm{SI}}^{2} \mathbf{I}_{\mathbf{N}}-\boldsymbol{\Omega}_{\mathrm{SI}}\right)$, respectively, where $\Omega_{\mathrm{da}}$ and $\boldsymbol{\Omega}_{\mathrm{SI}}$ are diagonal matrices with $k$-th diagonal element

$$
\begin{aligned}
\sigma_{\mathrm{da}, k}^{2} & =\frac{\tau \rho_{p} \beta_{\mathrm{da}, k}^{2}}{1+\tau \rho_{p} \beta_{\mathrm{da}, k}}, \\
\sigma_{\mathrm{SI}, k}^{2} & =\frac{\tau \rho_{p} \sigma_{\mathrm{SI}}^{4}}{1+\tau \rho_{p} \sigma_{\mathrm{SI}}^{2}},
\end{aligned}
$$

respectively, where $\rho_{p}$ denotes the transmit sinal-to-noise ratio (SNR) of each pilot symbol. By using the reciprocity properties of the wireless channel, the HAP-to-users CSI is obtained $\mathbf{b}$ as $\hat{\mathbf{G}}_{\mathrm{ad}}=\hat{\mathbf{G}}_{\mathrm{da}}^{T}\left(\sigma_{\mathrm{ad}, k}^{2}=\sigma_{\mathrm{da}, k}^{2}\right)$.

At time instant $i$, the received signal at the $k$-th $\mathrm{UE}$ is given by

$$
\begin{aligned}
y_{\mathrm{u}, k}[i]= & \sqrt{p_{\mathrm{a}}} \mathbf{g}_{\mathrm{au}, k} \mathbf{w}_{\mathrm{E}, k} s_{\mathrm{E}}[i]+\sqrt{p_{\mathrm{a}}} \sum_{\ell \neq k}^{K_{\mathrm{ul}}} \mathbf{g}_{\mathrm{au}, k} \mathbf{w}_{\mathrm{E}, \ell} s_{\mathrm{E}}[i] \\
& +\sqrt{p_{p}} \mathbf{g}_{\mathrm{du}, k} \mathbf{\Phi}_{\mathrm{d}, i}+n_{\mathrm{u}, k}[i],
\end{aligned}
$$

where $\mathbf{g}_{\mathrm{au}, k} \in \mathbb{C}^{1 \times N}$ and $\mathbf{g}_{\mathrm{du}, k} \in \mathbb{C}^{1 \times K_{\mathrm{dll}}}$ denote the channel vectors from the HAP and the $K_{\mathrm{dl}}$ CUs to the UE $k$, respectively. In particular, $\mathbf{g}_{\mathrm{au}, k}$ and $\mathbf{g}_{\mathrm{du}, k}$ can be expressed as $\mathbf{g}_{\mathrm{au}, k}=\sqrt{\beta_{\mathrm{au}, k}} \mathbf{h}_{\mathrm{au}, k}$ and $\mathbf{g}_{\mathrm{du}, k}=\mathbf{D}_{\mathrm{du}, k}^{1 / 2} \mathbf{h}_{\mathrm{du}, k}$, respectively, where $\mathbf{h}_{\mathrm{au}, k}$ and $\mathbf{h}_{\mathrm{du}, k}$ denote the small-scale fading vectors whose eateries are i.i.d. $\mathcal{C N}(0,1), \beta_{\mathrm{au}, k}$ is the large-scale fading coefficient between HAP and UE $k$, and $\mathbf{D}_{\mathrm{du}, k}$ is the large-scale fading diagonal matrix whose $m$-th diagonal elements, i.e., $\beta_{\mathrm{du}, k m}$, models the large-scale fading between the $m$-th $\mathrm{CU}$ and $k$-th $\mathrm{UE}, \boldsymbol{\Phi}_{\mathrm{d}, i}$ denotes the $i$-th column of matrix $\boldsymbol{\Phi}_{\mathrm{d}}$, and $n_{\mathrm{u}, k}[i] \sim \mathcal{C N}\left(0, \sigma_{n}^{2}\right)$ is the AWGN at the $k$-th UE.

The $k$-th UE rectifies the RF signal $y_{\mathrm{u}, k}[i]$ for charging its battery. We assume that the amount of the harvested energy from the received noise is negligible since the noise power is far less than the received power from the HAP. Thereby the noise power is neglected from the harvested energy [27], [28]. Moreover, since transmit power of the CUs' is low we assume that the amount of the harvested energy from the

\footnotetext{
${ }^{1}$ In order to minimize the MMSE estimation error $\boldsymbol{\Phi}_{\mathrm{E}}$ and $\boldsymbol{\Phi}_{\mathrm{d}}$ must be pairwisely orthogonal. This implies that $\tau \geq N+K_{\mathrm{dl}}$. Although this condition implies that the pilot resources needed to estimate the SI channel is high, in practice there is no need to increase the number of antennas beyond a certain value to achieve high spectral efficiency [25].
}

signal is relatively marginal. Therefore, transmit power of the $k$-th UE's during the consequent $(1-\alpha)$ fraction of the time frame can be written as

$$
\begin{aligned}
p_{\mathrm{u}, k} & =\kappa p_{\mathrm{a}} \sum_{j=1}^{K_{\mathrm{ul}}}\left|\mathbf{g}_{\mathrm{au}, k} \mathbf{w}_{\mathrm{E}, j}\right|^{2} \\
& =\kappa p_{\mathrm{a}} \beta_{\mathrm{au}, k} \mathbf{h}_{\mathrm{au}, k} \mathbf{W}_{\mathrm{E}} \mathbf{W}_{\mathrm{E}}^{\dagger} \mathbf{h}_{\mathrm{au}, k}^{\dagger},
\end{aligned}
$$

where $\mathbf{W}_{\mathrm{E}}=\left[\mathbf{w}_{\mathrm{E}, 1}, \cdots, \mathbf{w}_{\mathrm{E}, K_{\mathrm{ul}}}\right] \in \mathbb{C}^{N \times K_{\mathrm{ul}}}$ denotes the energy beamforming matrix, $\kappa=\frac{\eta \alpha}{1-\alpha}$ where $0<\eta<1$ denotes the energy conversion efficiency.

2) Uplink and Downlink Data Transmission: The HAP uses the estimates of the SI and HAP-to-CU channels to performs beamforming to transmit information to the users ${ }^{2}$. We assume that perfect CSI of the UE-to-HAP channels is known at the HAP. In general, UE positions are changing relatively slowly. Therefore, the HAP-to-UE channels can be accurately estimated. Moreover, the CSI can be updated using the subsequent UE to HAP transmissions in the uplink.

At time instant $i$ of the second phase, the HAP uses the receive combining matrix $\mathbf{W}_{r}=\left[\mathbf{w}_{r, 1}, \cdots, \mathbf{w}_{r, K_{\mathrm{ul}}}\right] \in$

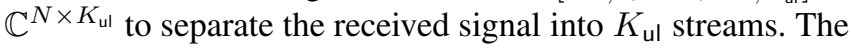
$k$-th column of $\mathbf{W}_{r}$, i.e., $\mathbf{w}_{r, k}$, is the receive beamformer corresponding to the $k$-th UE. Therefore, after applying the receive combining matrix $\mathbf{W}_{r}$, the received signal at the HAP is given by

$$
\mathbf{r}_{\mathrm{a}}[i]=\mathbf{W}_{r}^{\dagger} \mathbf{G}_{\mathrm{ua}} \mathbf{x}_{\mathrm{u}}[i]+\mathbf{W}_{r}^{\dagger} \mathbf{H}_{\mathrm{SI}}^{T} \hat{\mathbf{W}}_{t} \mathbf{x}_{\mathrm{d}}[i]+\mathbf{W}_{r}^{\dagger} \mathbf{n}_{\mathrm{a}}[i],
$$

where $\mathbf{G}_{\text {ua }}=\left[\mathbf{g}_{\text {ua }, 1}, \cdots, \mathbf{g}_{\text {ua }, K_{\text {ul }}}\right] \in \mathbb{C}^{N \times K_{\text {ul }}}$ (with $\mathbf{g}_{\text {ua }, k}=$ $\mathbf{g}_{\mathrm{au}, k}^{T}$ ) is the channel matrix from $K_{\mathrm{ul}}$ sensors to the HAP, $\mathbf{x}_{\mathrm{u}}[i]=\left[x_{\mathrm{u}, 1}[i], \cdots, x_{\mathrm{u}, K_{\mathrm{ul}}}[i]\right]^{T}$ with $\mathbf{Q}_{\mathrm{u}} \triangleq \mathbb{E}\left\{\mathbf{x}_{\mathrm{u}} \mathbf{x}_{\mathrm{u}}^{\dagger}\right\}=$ $\operatorname{diag}\left\{P_{\mathrm{u}, 1}, \cdots, P_{\mathrm{u}, K_{\mathrm{ul}}}\right\}$ and $\mathbf{x}_{\mathrm{d}}[i]=\left[x_{\mathrm{d}, 1}[i], \cdots, x_{\mathrm{d}, K_{\mathrm{dl}}}[i]\right]^{T}$ with $\mathbb{E}\left\{\mathbf{x}_{\mathrm{d}} \mathbf{x}_{\mathrm{d}}^{\dagger}\right\}=p_{\mathrm{a}} \mathbf{I}_{K_{\mathrm{dl}}}$ are the information signals corresponding to the UEs and CUs, respectively. Downlink beamformer at the HAP is denoted by $\hat{\mathbf{W}}_{t}=\left[\hat{\mathbf{w}}_{t, 1}, \cdots, \hat{\mathbf{w}}_{t, K_{\mathrm{dl}}}\right] \in$ $\mathbb{C}^{M \times K_{\mathrm{dl}}}$ in which the $k$-th column, i.e., $\hat{\mathbf{w}}_{t, k}$, is the transmit beamformer corresponding to the $k$-th $\mathrm{CU}$.

The received signal from the UE $k$, denoted by $r_{\mathrm{a}, k}[i]$, can be expressed as

$$
\begin{aligned}
r_{\mathrm{a}, k}[i]= & \mathbf{w}_{r, k}^{\dagger} \mathbf{g}_{\mathrm{ua}, k} x_{\mathbf{u}, k}[i]+\sum_{\ell \neq k}^{K_{\mathrm{ul}}} \mathbf{w}_{r, k}^{\dagger} \mathbf{g}_{\mathrm{ua}, \ell} x_{\mathbf{u}, \ell}[i] \\
& +\sum_{\ell=1}^{K_{\mathrm{dll}}} \mathbf{w}_{r, k}^{\dagger} \hat{\mathbf{H}}_{\mathrm{SI}}^{T} \hat{\mathbf{w}}_{t, \ell} x_{\mathrm{d}, \ell}[i]+\sum_{\ell=1}^{K_{\mathrm{dl}}} \mathbf{w}_{r, k}^{\dagger} \mathcal{E}_{\mathrm{SI}}^{T} \hat{\mathbf{w}}_{t, \ell} x_{\mathrm{d}, \ell}[i] \\
& +\mathbf{w}_{r, k}^{\dagger} \mathbf{n}_{\mathrm{a}}[i] .
\end{aligned}
$$

We note that the HAP knows its own transmit signals $\hat{\mathbf{w}}_{t, \ell} x_{\mathrm{d}, \ell}[i]$ and the MMSE estimate of the SI channel.

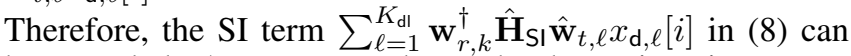
be canceled [5]. However, due to hardware impairments at the full-duplex transceiver, the SI term cannot be completely removed. We consider a more realistic case where SI cancellation is imperfect and residual interference exists. We model

\footnotetext{
${ }^{2}$ In massive MIMO systems, channel reciprocity is widely exploited to estimate the channel response on the uplink and then use the acquired CSI for transmit beamforming of payload data, provided that the system operates in TDD mode [29].
} 
the residual SI as $\tilde{\mathbf{H}}_{\mathrm{SI}} \sim \mathcal{C N}\left(0, k_{1} \sigma_{\mathrm{SI}}^{2}\right)$, where the parameter $k_{1}$ defines the level of residual interference. In particular, $k_{1}=0$ implies perfect interference cancellation [5]. Accordingly, denoting $\tilde{\mathcal{E}}_{\mathrm{SI}}=\mathcal{E}_{\mathrm{SI}}+\tilde{\mathbf{H}}_{\mathrm{SI}}$, the observed signal-tointerference-plus-noise ratio (SINR) at HAP corresponding to the UE $k$ can be written as

$\gamma_{\mathrm{a}, k}\left(\hat{\mathbf{W}}_{t}, \mathbf{w}_{r, k}, \mathbf{W}_{\mathrm{E}}, \alpha\right)=$

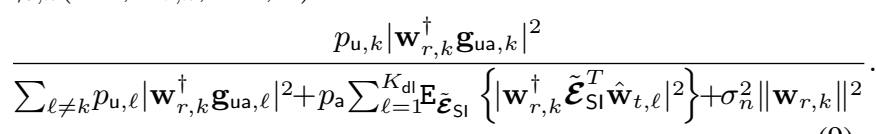

At time instant $i$, the received signal at all CUs can be expressed as

$$
\mathbf{r}_{\mathbf{d}}[i]=\mathbf{G}_{\mathbf{a d}} \hat{\mathbf{W}}_{t} \mathbf{x}_{\mathbf{d}}[i]+\mathbf{G}_{\mathbf{u d}} \mathbf{x}_{\mathbf{u}}[i]+\mathbf{n}_{\mathbf{d}}[i]
$$

where $\mathbf{G}_{\mathrm{ad}}=\mathbf{G}_{\mathrm{da}}^{T}, \mathbf{G}_{\mathrm{ud}}=\left[\mathbf{g}_{\mathrm{ud}, 1} ; \cdots ; \mathbf{g}_{\mathrm{ud}, K_{\mathrm{dl}}}\right] \in \mathbb{C}^{K_{\mathrm{dl}} \times K_{\mathrm{ul}}}$ represents channel matrix between the $K_{\mathrm{ul}}$ UEs and the $K_{\mathrm{dl}}$ CUs, i.e., $g_{\mathrm{ud}, k m}=\left[\mathbf{G}_{\mathrm{ud}}\right]_{k m}$ is the channel coefficient between the $m$-th UE and the $k$-th CU which can be written as $g_{\text {ud }, k m}=\sqrt{\beta_{\text {ud }, k m}} h_{\text {ud }, k m}$ where $h_{\text {ud }, k m}$ is the fast fading coefficient ( $\mathrm{g}_{\mathrm{ud}, k} \in \mathbb{C}^{1 \times K_{\mathrm{ul}}}$ is the channel vector from $K_{\mathrm{ul}}$ UEs to $k$-th $\mathrm{CU}) ; \mathbf{n}_{\mathrm{d}}[i]$ is the AWGN vector at $K_{\mathrm{dl}} \mathrm{CUs}$. According to (10), the received signal at the $k$-th $\mathrm{CU}$ is

$$
\begin{aligned}
r_{\mathrm{d}, k}[i]= & \mathbf{g}_{\mathrm{ad}, k} \hat{\mathbf{w}}_{t, k} x_{\mathrm{d}, k}[i]+\sum_{\ell \neq k}^{K_{\mathrm{dl}}} \mathbf{g}_{\mathrm{ad}, k} \hat{\mathbf{w}}_{t, \ell} x_{\mathrm{d}, \ell}[i] \\
& +\mathbf{g}_{\mathrm{ud}, k} \mathbf{x}_{\mathrm{u}}[i]+n_{\mathrm{d}, k}[i], \\
= & \hat{\mathbf{g}}_{\mathrm{ad}, k} \hat{\mathbf{w}}_{t, k} x_{\mathrm{d}, k}[i]+\sum_{\ell \neq k}^{K_{\mathrm{dl}}} \hat{\mathbf{g}}_{\mathrm{ad}, k} \hat{\mathbf{w}}_{t, \ell} x_{\mathrm{d}, \ell}[i] \\
& +\sum_{\ell=1}^{K_{\mathrm{dl}}} \varepsilon_{\mathrm{ad}, k} \hat{\mathbf{w}}_{t, \ell} x_{\mathrm{d}, \ell}[i]+\mathbf{g}_{\mathrm{ud}, k} \mathbf{x}_{\mathbf{u}}[i]+n_{\mathrm{d}, k}[i],
\end{aligned}
$$

where $n_{\mathrm{d}, k}[i]$ is the $k$-th element of $\mathbf{n}_{\mathrm{d}}[i]$ and $\varepsilon_{\mathrm{ad}, k}$ is the $k$ th column of $\mathcal{E}_{\text {ad }}\left(\mathcal{E}_{\text {ad }}=\mathcal{E}_{\mathrm{da}}^{T}\right)$. We assume that the effective channel gains, i.e., $\left|\hat{\mathbf{g}}_{\mathrm{ad}, k} \hat{\mathbf{w}}_{t, k}\right|^{2}$ is sent to the $k$-th user via a low-rate feedback link. Moreover, the $k$-th CU never knows the channel estimation error $\varepsilon_{\mathrm{ad}, k}$ and $g_{\mathrm{ud}, k \ell}$. Therefore, from (11) the SINR at the $k$-th CU can be expressed as (12) at the top of the next page.

\section{Achievable Rate Analysis}

In this section, we derive the uplink and downlink achievable rates for MRC/MRT and $\mathrm{ZF}$ processing at the $\mathrm{HAP}^{3}$. We assume that HAP deploys MRT beamformer for energy transfer to UEs as motivated in [15]. Therefore, the

\footnotetext{
${ }^{3}$ Among different transmit/receive beamforming schemes, MRC/MRT and $\mathrm{ZF}$ are two simple linear and practical candidates for massive MIMO systems, which are widely used in the literature [2], [30], [31]. We notice that, a major concern about deployment of massive MIMO technology is how operations on large matrices and the interconnection of the many antenna signals can be efficiently performed in real-time. Digital signal processing required to realize the massive MIMO system concept has been investigated in [32], where the co-design of algorithms, hardware architecture, and circuits have been examined in detail. Specifically, it has been shown in [32] that, low-complexity digital circuitry implementations in deeply scaled silicon are possible, despite the excess number of antenna signals.
}

energy beamformer is set to $\mathbf{w}_{\mathrm{E}, k}=\frac{\mathbf{g}_{\mathrm{au}, k}^{\dagger}}{\left\|\mathbf{g}_{\mathrm{au}, k}\right\|}$. The achievable downlink and uplink sum-rate of the system are respectively given by

$$
\begin{aligned}
& R_{\mathrm{U}}=(1-\alpha) \sum_{k=1}^{K_{\mathrm{ul}}} \mathrm{E}\left\{\log _{2}\left(1+\gamma_{\mathrm{a}, k}\left(\hat{\mathbf{W}}_{t}, \mathbf{W}_{r}, \mathbf{W}_{\mathrm{E}}, \alpha\right)\right)\right\}, \\
& R_{\mathrm{D}}=(1-\alpha) \sum_{k=1}^{K_{\mathrm{dll}}} \mathrm{E}\left\{\log _{2}\left(1+\gamma_{\mathrm{d}, k}\left(\hat{\mathbf{W}}_{t}, \mathbf{W}_{\mathrm{E}}, \alpha\right)\right)\right\} .
\end{aligned}
$$

\section{A. MRC/MRT Processing}

We consider MRC/MRT processing since it is a very simple signal processing architecture. The MRC/MRT processing maximizes the received SNRs, while neglect the inter-user interference and SI effect. Therefore, MRC/MRT processing provides good performance at low SNRs, and works poorly at high SNRs. For the MRC/MRT scheme, the receive and transmit beamformers are given by

$$
\begin{aligned}
& \mathbf{W}_{r}^{\mathrm{MRC}}=\alpha_{\mathrm{MRC}} \mathbf{G}_{\mathrm{ua}}, \\
& \hat{\mathbf{W}}_{t}^{\mathrm{MRT}}=\alpha_{\mathrm{MRT}} \hat{\mathbf{G}}_{\mathrm{ad}}^{\dagger},
\end{aligned}
$$

where $\alpha_{\mathrm{MRC}}$ and $\alpha_{\mathrm{MRT}}$ are normalization factors, chosen to satisfy a long-term total transmit power constraint at the HAP, given by [33]

$$
\begin{aligned}
& \alpha_{\mathrm{MRC}}=1 / \sqrt{\mathrm{E}\left\{\operatorname{tr}\left(\mathbf{G}_{\mathrm{ua}}^{\dagger} \mathbf{G}_{\mathrm{ua}}\right)\right\}}=\sqrt{\frac{1}{N \sum_{k=1}^{K_{\mathrm{ul}}} \beta_{\mathrm{au}, k}}}, \\
& \alpha_{\mathrm{MRT}}=1 / \sqrt{\mathrm{E}\left\{\operatorname{tr}\left(\hat{\mathbf{G}}_{\mathrm{ad}} \hat{\mathbf{G}}_{\mathrm{ad}}^{\dagger}\right)\right\}}=\sqrt{\frac{1}{M \sum_{k=1}^{K_{\mathrm{dl}}} \sigma_{\mathrm{ad}, k}^{2}}} .
\end{aligned}
$$

We next derive new approximate closed-form expressions for the achievable uplink and downlink rates of the system with MRC/MRT processing.

Proposition 1: With MRC/MRT processing at the HAP, achievable uplink rate of the $k$-th UE can be approximated as

$$
\begin{aligned}
R_{\mathrm{ul}, \mathrm{k}}^{\mathrm{MR}} \approx(1-\alpha) & \int_{0}^{\infty}\left(1-\left(\frac{1}{1+\varphi_{k} z}\right)^{N}\right) \\
& \times \prod_{\ell=1, \ell \neq k}^{K_{\mathrm{ul}}}\left(\frac{1}{1+\varphi_{\ell} z}\right) \frac{e^{-\psi_{\mathrm{u}} z}}{z} d z,
\end{aligned}
$$

where $\varphi_{j}=\kappa \beta_{\mathrm{au}, j}\left(K_{\mathrm{ul}}+N-1\right)$ with $j \in\{k, \ell\}$ and $\psi_{\mathrm{u}}=$ $k_{1} \sigma_{\mathrm{SI}}^{2}+\frac{\sigma_{\mathrm{SI}}^{2}}{1+\tau p_{\mathrm{a}} \sigma_{\mathrm{SI}}^{2}}+\frac{1}{\rho_{\mathrm{a}}}$, where $\rho_{\mathrm{a}}=\frac{p_{\mathrm{a}}}{\sigma_{n}^{2}}$.

Proof: See Appendix A.

Although the integral in (16) seems not to admit a closedform solution, it can be efficiently evaluated numerically using Matlab or Mathematica. Alternatively, in the following we derive a closed-form lower bound for the uplink achievable rate of the system with MRC/MRT processing.

Proposition 2: With MRC/MRT processing at the HAP, the uplink achievable rate of the $k$-th UE can be lower bounded as $R_{\mathrm{ul}, \mathrm{k}}^{\mathrm{MR}} \geq \tilde{R}_{\mathrm{ul}, \mathrm{k}}^{\mathrm{MR}}=(1-\alpha) \log _{2}(1+$ 


$$
\gamma_{\mathrm{d}, k}\left(\hat{\mathbf{W}}_{t}, \mathbf{W}_{\mathrm{E}}, \alpha\right)=\frac{p_{\mathrm{a}}\left|\hat{\mathbf{g}}_{\mathrm{ad}, k} \hat{\mathbf{w}}_{t, k}\right|^{2}}{p_{\mathrm{a}} \sum_{\ell \neq k}^{K_{\mathrm{dll}}}\left|\hat{\mathbf{g}}_{\mathrm{ad}, k} \hat{\mathbf{w}}_{t, \ell}\right|^{2}+p_{\mathrm{a}} \sum_{\ell=1}^{K_{\mathrm{dl}}} \mathrm{E}_{\varepsilon_{\mathrm{ad}, k}}\left\{\left|\varepsilon_{\mathrm{ad}, k} \hat{\mathbf{w}}_{t, \ell}\right|^{2}\right\}+\sum_{l=1}^{K_{\mathrm{ul}}} p_{\mathrm{u}, \ell} \mathrm{E}\left\{\left|g_{\mathrm{ud}, k \ell}\right|^{2}\right\}+\sigma_{n}^{2}} .
$$

$$
R_{\mathrm{dl}, \mathrm{k}}^{\mathrm{MR}} \approx \tilde{R}_{\mathrm{dl}, \mathrm{k}}^{\mathrm{MR}}=(1-\alpha) \log _{2}\left(1+\frac{M \beta_{\mathrm{ad}, k}}{\sum_{\ell=1, \ell \neq k}^{K_{\mathrm{dl}}} \beta_{\mathrm{ad}, \ell}+\sum_{\ell=1}^{K_{\mathrm{dl}}}\left(\frac{\beta_{\mathrm{ad}, \ell}}{\beta_{\mathrm{ad}, k}}\right)\left(\kappa\left(K_{\mathrm{ul}}+N-1\right) \sum_{l=1}^{K_{\mathrm{ul}}} \beta_{\mathrm{ud}, k \ell} \beta_{\mathrm{au}, \ell}+\frac{1}{\rho_{\mathrm{a}}}\right)}\right) .
$$

$$
\left.\frac{\kappa \beta_{\mathrm{au}, k}^{2}\left(K_{\mathrm{ul}}+N-1\right)(N-1)}{\kappa \sum_{\ell \neq k}^{K_{\mathrm{ul}}} \beta_{\mathrm{au}, \ell}^{2}\left(K_{\mathrm{ul}}+N-1\right)+k_{1} \sigma_{\mathrm{Sl}}^{2}+\frac{\sigma_{\mathrm{Sl}}^{2}}{1+\tau p_{\mathrm{a}} \sigma_{\mathrm{Sl}}^{2}}+\frac{1}{\rho_{\mathrm{a}}}}\right) .
$$

Proof: See Appendix B.

Remark 1: The aforementioned result in (17) indicates that, the SI can be mitigated by using massive transmit antennas at the HAP. Furthermore, the intra-sensor interference is vanished. [9]

Corollary 1: With MRC/MRT processing and genie aided receiver at the HAP, a lower bound on the achievable uplink rate of the $k$-th UE is given by

$$
\begin{aligned}
R_{\mathrm{ul}, \mathrm{k}}^{\mathrm{MR}} \geq \tilde{R}_{\mathrm{ul}, \mathrm{k}}^{\mathrm{MR}}=(1-\alpha) \log _{2}(1+ & \left.\frac{\kappa \beta_{\mathrm{au}, k}^{2}\left(K_{\mathrm{ul}}+N-1\right)(N-1)}{\kappa \sum_{\ell \neq k}^{K} \beta_{\mathrm{au}, \ell}^{2}\left(K_{\mathrm{ul}}+N-1\right)+k_{1} \sigma_{\mathrm{Sl}}^{2}+\frac{1}{\rho_{\mathrm{a}}}}\right) .
\end{aligned}
$$

Proof: See Appendix C.

Now we turn our attention to derive the downlink achievable rate of the MRC/MRT processing. The following proposition provides the analytical expressions corresponding to the downlink achievable rate.

Proposition 3: The achievable downlink rate of the $k$-th $\mathrm{CU}$ with MRC/MRT processing can be approximated by

$$
\begin{aligned}
& R_{\mathrm{dl}, \mathrm{k}}^{\mathrm{MR}} \approx \tilde{R}_{\mathrm{dl}, \mathrm{k}}^{\mathrm{MR}}=(1-\alpha) \log _{2}(1+ \\
& \left.\frac{M \sigma_{\mathrm{ad}, k}^{2}}{\sum_{\ell=1, \ell \neq k}^{K_{\mathrm{dl}}} \sigma_{\mathrm{ad}, \ell}^{2}+\left(\beta_{\mathrm{ad}, k}-\sigma_{\mathrm{ad}, k}^{2}+\Gamma\right) \sum_{\ell=1}^{K_{\mathrm{dl}}}\left(\frac{\sigma_{\mathrm{ad}, \ell}^{2}}{\sigma_{\mathrm{ad}, k}^{2}}\right)}\right),
\end{aligned}
$$

where $\Gamma=\kappa\left(K_{\mathrm{ul}}+N-1\right) \sum_{\ell=1}^{K_{\mathrm{ul}}} \beta_{\mathrm{ud}, k \ell} \beta_{\mathrm{au}, \ell}+\frac{1}{\rho_{\mathrm{a}}}$.

Proof: See Appendix D.

It can be readily inferred from Proposition 3 that the achievable downlink rate of the $k$-th CU with MRC/MRT processing is directly proportional to the number of transmit antennas at the HAP, while it is in inverse ratio with the number of receive antennas and the value of $K_{\mathrm{ul}}$ and $K_{\mathrm{dl}}$.

Remark 2: The achievable rate in (19) is obtained by using the approximation $\mathrm{E}\left\{\log _{2}\left(1+\frac{X}{Y}\right)\right\} \approx$ $\log _{2}\left(1+\frac{E\{X\}}{E\{Y\}}\right)[34$, Lemma 1]. Following the proof of the lemma in [34, Appendix I], if $X$ and $Y$ are two non-negative RVs with properties that $\frac{\operatorname{VAR}(Y)}{\mathrm{E}^{2}(Y)}$ and $\frac{\operatorname{VAR}(X+Y)}{\mathrm{E}^{2}(X+Y)}$ approaches zero, the approximation is asymptotically tight. Here, we can readily check that $\frac{\operatorname{VAR}(Y)}{\mathrm{E}^{2}(Y)} \rightarrow 0$ and $\frac{\operatorname{VAR}(X+Y)}{\mathrm{E}^{2}(X+Y)} \rightarrow 0$ as $M \rightarrow \infty$, since the two numerators increase with order $M$ while the two denominators increase with order $M^{2}$. Hence, the approximation is asymptotically tight as $M$ increases.

Corollary 2: The achievable downlink rate of the genie receiver with MRC/MRT processing and for $M \geq 2$ can be approximated as (20) at the top of the page.

Proof: Proof follows similar steps to that of Proposition 3.

\section{B. ZF Processing}

In this scheme, ZF receiver and ZF precoding are deployed at the HAP. Moreover, by projecting each stream onto the orthogonal complement of the interpair interference, the interpair interferences are nulled out. In particular, the interpair uplink interference in (8) is represented by the term $\sum_{\ell \neq k}^{K_{\mathrm{ul}}} \mathbf{w}_{r, k}^{\dagger} \mathbf{g}_{\mathrm{ua}, \ell} x_{\mathbf{u}, \ell}[i]$, while the term $\sum_{\ell \neq k}^{K_{\mathrm{dl}}} \mathbf{g}_{\mathrm{ad}, k} \hat{\mathbf{w}}_{t, \ell} x_{\mathrm{d}, \ell}[i]$ in (11) represents the interpair downlink interference. The $\mathrm{ZF}$ receiver and ZF precoding matrices are respectively given by [9]

$$
\begin{aligned}
& \mathbf{W}_{r}^{\mathrm{ZF}}=\alpha_{\mathrm{RZF}} \mathbf{G}_{\text {ua }}\left(\mathbf{G}_{\mathrm{ua}}^{\dagger} \mathbf{G}_{\text {ua }}\right)^{-1}, \\
& \hat{\mathbf{W}}_{t}^{\mathrm{ZF}}=\alpha_{\mathrm{TZF}} \hat{\mathbf{G}}_{\mathrm{ad}}^{\dagger}\left(\hat{\mathbf{G}}_{\mathrm{ad}} \hat{\mathbf{G}}_{\mathrm{ad}}^{\dagger}\right)^{-1},
\end{aligned}
$$

where $\alpha_{\text {RZF }}$ and $\alpha_{\text {TZF }}$ are normalization constants satisfying long-term total transmit power constraint at the HAP and given by [33]

$$
\begin{aligned}
& \alpha_{\mathrm{RZF}}=1 / \sqrt{\mathrm{E}\left\{\operatorname{tr}\left(\mathbf{G}_{\mathrm{ua}}^{\dagger} \mathbf{G}_{\mathrm{ua}}\right)^{-1}\right\}}=\sqrt{\frac{N-K_{\mathrm{ul}}}{\sum_{k=1}^{K_{\mathrm{ul}}} \beta_{\mathrm{au}, k}^{-1}}}, \\
& \alpha_{\mathrm{TZF}}=1 / \sqrt{\mathrm{E}\left\{\operatorname{tr}\left(\hat{\mathbf{G}}_{\mathrm{ad}} \hat{\mathbf{G}}_{\mathrm{ad}}^{\dagger}\right)^{-1}\right\}}=\sqrt{\frac{M-K_{\mathrm{dl}}}{\sum_{k=1}^{K_{\mathrm{dl}}} \sigma_{\mathrm{ad}, k}^{-2}}} .
\end{aligned}
$$

In the sequel, we provide a new approximate closed-form expressions for the lower bounds of uplink and downlink achievable rates for ZF processing. When the number of UEs and/or CUs is high, the computational complexity of the ZF precoder lies in the inversion of large scale matrices. A lowcomplexity method based on matrix inversion is proposed in [35] to decrease the computational complexity of the ZF and the MMSE precoders.

Proposition 4: With ZF processing and $M \geq K_{\mathrm{dl}}, N \geq$ $K_{\mathrm{ul}}$, the uplink achievable rate from the $k$-th UE can be lower bounded as

$R_{\mathrm{ul}, \mathrm{k}}^{\mathrm{ZF}} \geq \tilde{R}_{\mathrm{ul}, \mathrm{k}}^{\mathrm{ZF}}=(1-\alpha) \log _{2}\left(1+\frac{\kappa \beta_{\mathrm{au}, k}^{2}\left(N^{2}-K_{\mathrm{ul}}^{2}\right)}{k_{1} \sigma_{\mathrm{Sl}}^{2}+\frac{\sigma_{\mathrm{Sl}}^{2}}{1+\tau p_{\mathrm{a}} \sigma_{\mathrm{Sl}}^{2}}+\frac{1}{\rho_{\mathrm{a}}}}\right)$

Proof: See Appendix E. 
Proposition 4 indicates that, the uplink achievable rate from the $k$-th UE with ZF processing increases with the number of HAP transmit antennas unboundedly and decrease with the number of UEs. Moreover, comparison between (17) and (23) reveals that by increasing the number of HAP transmit antennas, the uplink achievable rate of $\mathrm{ZF}$ processing is more pronounced than the MRC/MRT processing.

Proposition 5: With ZF processing the downlink achievable rate of the $k$-th $\mathrm{CU}$ can be approximated as

$$
\begin{aligned}
& R_{\mathrm{dl}, \mathrm{k}}^{\mathrm{ZF}} \approx(1-\alpha) \log _{2}(1+\left(M-K_{\mathrm{dl}}\right) / \sum_{\ell=1}^{K_{\mathrm{dl}}} \sigma_{\mathrm{ad}, \ell}^{-2} \\
&\left.\frac{\left(\beta_{\mathrm{ad}, k}-\sigma_{\mathrm{ad}, k}^{2}\right)+\kappa\left(K_{\mathrm{ul}}+N-1\right) \sum_{l=1}^{K_{\mathrm{ul}}} \beta_{\mathrm{au}, \ell} \beta_{\mathrm{ud}, k \ell}+1 / \rho_{\mathrm{a}}}{2}\right) .
\end{aligned}
$$

Proof: The proof is omitted due to its similarity to the proof of Proposition 4.

Proposition 5 implies that the downlink achievable rate of the $k$-th $\mathrm{CU}$ is a decreasing function of the number of CUs and UEs. This is due to the fact that, by increasing the number of CUs and UEs, the inter-user interference and intra-sensor interference are increased.

In order to investigate the potential for power saving in the data transmission phase, we set $P_{a}=\frac{E_{a}}{N^{2}}$, where $E_{a}$ is fixed regardless of $N$. When $N \rightarrow \infty$, the asymptotic performances of MRC/MRT and ZF processing are the same and given by

$$
\tilde{R}_{\mathrm{ul}, \mathrm{k}}^{\mathrm{ZF}}=\tilde{R}_{\mathrm{ul}, \mathrm{k}}^{\mathrm{MR}} \rightarrow(1-\alpha) \log _{2}\left(1+\frac{E_{\mathrm{a}}}{\sigma_{n}^{2}} \kappa \beta_{\mathrm{au}, k}^{2}\right), N \rightarrow \infty .
$$

This result shows that, with large antenna arrays, we can reduce the transmitted power of the HAP proportionally to $1 / N^{2}$. The same results have been found in the literature, in context of multipair FD relaying [9] and uplink massive MIMO systems [31].

Remark 3: Comparing $\tilde{R}_{\mathrm{dl}, \mathrm{k}}^{\mathrm{MR}}$ in (19) with $R_{\mathrm{dl}, \mathrm{k}}^{\mathrm{ZF}}$ in (24), the downlink gain achieved by using ZF processing over MRC/MRT processing at the $k$-th $\mathrm{CU}$ and for large transmit antenna arrays, $M$, can be quantified as

$$
G_{\mathrm{d}}^{k} \approx \log _{2}\left(\frac{1}{\sigma_{\mathrm{ad}, k}^{4}} \frac{\sum_{\ell=1}^{K_{\mathrm{dl}}} \sigma_{\mathrm{ad}, \ell}^{2}}{\sum_{\ell=1}^{K_{\mathrm{dl}}} \sigma_{\mathrm{ad}, \ell}^{-2}}\right)
$$

The aim of using MRT energy beamformer in the proposed MRC/MRT and ZF processing schemes is to maximize the amount of power transferred from HAP to UEs. However, if UEs harvest high amount of energy during the energy harvesting phase, this might cause that the amount of interference inflicted on the CUs is significantly increased. Therefore, this motivates us to design the energy beamformers as well as energy harvesting time in order to establish a tradeoff between energy deliver to UEs and the interference they generate to downlink transmission from HAP.

\section{ENERGY BEAMFORMING OPTIMIZATION}

In this section, we consider joint optimization of energy beamformers at the HAP and time-split parameter. We aim to jointly design time-split parameter, receiver combiner, transmit and energy beamformers so that the sum downlink instantaneous spectral efficiency is maximized, while a predefined sum uplink instantaneous spectral efficiency at the HAP is ensured. Therefore, the optimization problem can be formulated as

$$
\begin{array}{cl}
\max _{\hat{\mathbf{W}}_{t}, \mathbf{W}_{r}, \mathbf{W}_{\mathrm{E}}, \alpha} & R_{\mathrm{D}}\left(\hat{\mathbf{W}}_{t}, \mathbf{W}_{\mathrm{E}}, \alpha\right) \\
\text { s.t } & R_{\mathrm{U}}\left(\hat{\mathbf{W}}_{t}, \mathbf{W}_{r}, \mathbf{W}_{\mathrm{E}}, \alpha\right) \geq \bar{R}_{\mathrm{u}} \\
& \left\|\hat{\mathbf{w}}_{t, k}\right\|=1, \quad k=1, \cdots, K_{\mathrm{dl}} \\
& \left\|\mathbf{w}_{r, k}\right\|=1, \quad k=1, \cdots, K_{\mathrm{ul}} \\
& \left\|\mathbf{w}_{\mathrm{E}, k}\right\|=1, \quad k=1, \cdots, K_{\mathrm{ul}}, \\
& 0 \leq \alpha \leq 1,
\end{array}
$$

where $\bar{R}_{\mathrm{u}}$ is the predefined rate for the uplink sum-rate. By inspecting the optimization problem (26), we can see that it is a complicated non-convex optimization problem with respect to (w.r.t) the receive/transmit beamforming vectors, energy beamforming vector, and time-split parameter $\alpha$. In order to circumvent this issue, by noticing that $\mathrm{ZF}$ and MRC/MRT processing schemes provide good performance and low complexity in massive MIMO systems, we proceed to develop an optimum energy beamforming and time-split parameter design. The optimization problem (26) with the $\mathrm{ZF}$ and MRC/MRT processing can be expressed as ${ }^{4}$

$$
\begin{array}{cl}
\max _{\mathbf{W}_{\mathrm{E}}, 0 \leq \alpha \leq 1} & R_{\mathrm{D}}^{i}\left(\mathbf{W}_{\mathrm{E}}, \alpha\right) \\
\text { s.t } & R_{\mathrm{U}}^{i}\left(\mathbf{W}_{\mathrm{E}}, \alpha\right) \geq \bar{R}_{\mathrm{u}} \\
& \left\|\mathbf{w}_{\mathrm{E}, k}\right\|=1, \quad k=1, \cdots, K_{\mathrm{ul}},
\end{array}
$$

where $i \in\{\mathrm{MR}, \mathrm{ZF}\}$.

The following proposition provides the closed-form approximate expressions for the downlink and uplink spectral efficiencies for problem (27) with energy beamforming matrix $\mathbf{W}_{\mathrm{E}}$.

Proposition 6: The downlink and uplink spectral efficiency of the system with MRC/MRT and ZF processing can be approximated as

$$
\begin{aligned}
R_{\mathrm{D}}^{i}\left(\mathbf{W}_{\mathrm{E}}, \alpha\right) \approx(1-\alpha) \sum_{k=1}^{K_{\mathrm{dl}}} \log _{2}(1+ \\
\left.\frac{\Lambda^{i}(\alpha)}{\frac{\eta \alpha}{(1-\alpha)} \operatorname{tr}\left(\mathbf{D}_{k} \mathbf{H}_{\mathrm{au}} \mathbf{W}_{\mathrm{E}} \mathbf{W}_{\mathrm{E}}^{\dagger} \mathbf{H}_{\mathrm{au}}^{\dagger} \mathbf{D}_{k}^{\dagger}\right)+\Phi_{k}^{i}(\alpha)}\right),
\end{aligned}
$$

and

$R_{\mathrm{U}}^{i}\left(\mathbf{W}_{\mathrm{E}}, \alpha\right) \approx(1-\alpha) \sum_{k=1}^{K_{\mathrm{ul}}} \log _{2}\left(1+\Psi_{k}^{i}(\alpha) \mathbf{h}_{\mathrm{au}, k} \mathbf{W}_{\mathrm{E}} \mathbf{W}_{\mathrm{E}}^{\dagger} \mathbf{h}_{\mathrm{au}, k}^{\dagger}\right)$,

respectively, where

$$
\mathbf{D}_{k}=\operatorname{diag}\left\{\sqrt{\beta_{\mathrm{ud}, k 1} \beta_{\mathrm{au}, 1}}, \cdots, \sqrt{\beta_{\mathrm{ud}, k K_{\mathrm{ul}}} \beta_{\mathrm{au}, K_{\mathrm{ul}}}}\right\}
$$

\footnotetext{
${ }^{4}$ The optimization problem (27) is a suboptimal problem of (26). However, since in in massive MIMO, the performance of ZF is very close to the performance of the optimal processing [36], it is expected that (27) provides a very good result which is close to the one obtained from (26).
} 


$$
\begin{aligned}
& \tilde{\mathbf{H}}_{\mathrm{au}, k}=\left[\mathbf{h}_{\mathrm{au}, 1}, \cdots, \mathbf{h}_{\left.\mathrm{au}, K_{\mathrm{ul}}\right]} \in \mathbb{C}^{K_{\mathrm{ul}} \times N}\right. \\
& \Lambda^{\mathrm{MR}}(\alpha)=\frac{M \sigma_{\mathrm{ad}, k}^{4}}{\sum_{\ell=1}^{K_{\mathrm{dl}}} \sigma_{\mathrm{ad}, \ell}^{2}}, \\
& \Phi_{k}^{\mathrm{MR}}(\alpha)=\sigma_{\mathrm{ad}, k}^{2} \frac{\sum_{\ell \neq k}^{K_{\mathrm{dl}}} \sigma_{\mathrm{ad}, \ell}^{2}}{\sum_{\ell=1}^{K_{\mathrm{dl}}} \sigma_{\mathrm{ad}, \ell}^{2}}+\left(\beta_{\mathrm{ad}, k}-\sigma_{\mathrm{ad}, k}^{2}+\frac{1}{\rho_{\mathrm{a}}}\right) \\
& \Psi_{k}^{\mathrm{MR}}(\alpha)=\frac{\alpha \eta N \beta_{\mathrm{au}, k}^{2}}{(1-\alpha)}\left(k_{1} \sigma_{\mathrm{SI}}^{2}+\frac{1}{1+\alpha p_{\mathrm{a}} \sigma_{\mathrm{SI}}^{2}}+\frac{1}{\rho_{\mathrm{a}}}\right)^{-1}, \\
& \Lambda^{\mathrm{ZF}}(\alpha)=\frac{\left(M-K_{\mathrm{dl}}\right)}{\sum_{\ell=1}^{K_{\mathrm{ul}}} \sigma_{\mathrm{ad}, \ell}^{-2}} \\
& \Phi_{k}^{\mathrm{ZF}}(\alpha)=\left(\beta_{\mathrm{ad}, k}-\sigma_{\mathrm{ad}, k}^{2}+\frac{1}{\rho_{\mathrm{a}}}\right) \\
& \Psi_{k}^{\mathrm{ZF}}(\alpha)=\frac{\alpha \eta \beta_{\mathrm{au}, k}}{(1-\alpha)}\left(k_{1} \sigma_{\mathrm{SI}}^{2}+\frac{1}{1+\alpha p_{\mathrm{a}} \sigma_{\mathrm{Sl}}^{2}}+\frac{1}{\rho_{\mathrm{a}}}\right)^{-1}
\end{aligned}
$$

with $\sigma_{\mathrm{ad}, k}^{2}=\alpha \beta_{\mathrm{ad}, k}\left(\alpha+\frac{1}{p_{p} \beta_{\mathrm{ad}, k}}\right)^{-1}$.

Proof: The proof follows simply from results developed in Proposition 3 and 4.

Let us define $\tilde{\mathbf{H}}_{\mathrm{au}, k}=\mathbf{D}_{k} \mathbf{H}_{\mathrm{au}}$. By invoking Proposition 6, the problem (27) can be written as

$$
\begin{array}{r}
\max _{\mathbf{W}_{\mathrm{E}, 0 \leq \alpha \leq 1}}(1-\alpha) \sum_{k=1}^{K_{\mathrm{dl}}} \log _{2}(1+ \\
\left.\frac{\Lambda^{i}(\alpha)}{\frac{\eta \alpha}{(1-\alpha)} \operatorname{tr}\left(\tilde{\mathbf{H}}_{\mathrm{au}, k} \mathbf{W}_{\mathrm{E}} \mathbf{W}_{\mathrm{E}}^{\dagger} \tilde{\mathbf{H}}_{\mathrm{au}, k}^{\dagger}\right)+\Phi_{k}^{i}(\alpha)}\right),(31 \mathrm{a}) \\
\text { s.t } \sum_{k=1}^{K_{\mathrm{ul}}} \log _{2}\left(1+\Psi_{k}^{i}(\alpha) \mathbf{h}_{\mathrm{au}, k} \mathbf{W}_{\mathrm{E}} \mathbf{W}_{\mathrm{E}}^{\dagger} \mathbf{h}_{\mathrm{au}, k}^{\dagger}\right) \geq \frac{\bar{R}_{\mathrm{u}}}{(1-\alpha)}, \\
\left\|\mathbf{w}_{\mathrm{E}, k}\right\|=1, \quad k=1, \cdots, K_{\mathrm{ul}} .
\end{array}
$$

Problem (31) is non-convex and finding its global optimum solutions is often computationally infeasible. In order to tackle the con-convexity of (31), we reformulate it by introducing an auxiliary variable, $\frac{1}{\tau_{k}}=$ $\frac{\eta \alpha}{(1-\alpha)} \operatorname{tr}\left(\tilde{\mathbf{H}}_{\mathrm{au}, k} \mathbf{W}_{\mathrm{E}} \mathbf{W}_{\mathrm{E}}^{\dagger} \tilde{\mathbf{H}}_{\mathrm{au}, k}^{\dagger}\right)+\Phi_{k}^{i}(\alpha)$, and then using

$$
\mathbf{h}_{\mathrm{au}, k} \mathbf{W}_{\mathrm{E}} \mathbf{W}_{\mathrm{E}}^{\dagger} \mathbf{h}_{\mathrm{au}, k}^{\dagger}=\overline{\mathbf{w}}_{\mathrm{E}}^{\dagger}\left(\mathbf{h}_{\mathrm{au}, k}^{\dagger} \mathbf{h}_{\mathrm{au}, k} \otimes \mathbf{I}_{K_{\mathrm{ul}}}\right) \overline{\mathbf{w}}_{\mathrm{E}},
$$

and

$$
\operatorname{tr}\left(\tilde{\mathbf{H}}_{\mathrm{au}, k} \mathbf{W}_{\mathrm{E}} \mathbf{W}_{\mathrm{E}}^{\dagger} \tilde{\mathbf{H}}_{\mathrm{au}, k}^{\dagger}\right)=\overline{\mathbf{w}}_{\mathrm{E}}^{\dagger}\left(\tilde{\mathbf{H}}_{\mathrm{au}, k}^{\dagger} \tilde{\mathbf{H}}_{\mathrm{au}, k} \otimes \mathbf{I}_{K_{\mathrm{ul}}}\right) \overline{\mathbf{w}}_{\mathrm{E}} .
$$

where $\overline{\mathbf{w}}_{\mathrm{E}}=\operatorname{vec}\left(\mathbf{W}_{\mathrm{E}}\right)$ and we used the fact that $\operatorname{tr}(\mathbf{A B C})=\operatorname{tr}(\mathbf{C A B})$ and $\operatorname{tr}(\mathbf{A X B})=\left(\mathbf{B}^{T} \otimes\right.$ $\mathbf{A}) \operatorname{vec}(\mathbf{X})$ [37]. Therefore, we rewrite (31) as

$$
\begin{gathered}
\max _{\overline{\mathbf{w}}_{\mathrm{E}}, \tau_{k}, 0 \leq \alpha \leq 1}(1-\alpha) \sum_{k=1}^{K_{\mathrm{dl}}} \log _{2}\left(1+\Lambda^{i}(\alpha) \tau_{k}\right), \\
\text { s.t } \quad \frac{\alpha \eta}{(1-\alpha)} \operatorname{tr}\left(\overline{\mathbf{W}}_{\mathrm{E}} \overline{\mathbf{A}}_{k}\right)+\Phi_{k}^{i}(\alpha) \leqslant \frac{1}{\tau_{k}}, \\
\forall k \in\left\{1, \cdots, K_{\mathrm{dl}}\right\},
\end{gathered}
$$

$$
\begin{aligned}
\sum_{k=1}^{K_{\mathrm{ul}}} & \log _{2}\left(1+\Psi_{k}^{i}(\alpha) \operatorname{tr}\left(\overline{\mathbf{W}}_{\mathrm{E}} \overline{\mathbf{B}}_{k}\right)\right) \geq \frac{\bar{R}_{\mathrm{u}}}{(1-\alpha)}, \\
& \operatorname{tr}\left(\overline{\mathbf{W}}_{\mathrm{E}}\right)=K_{\mathrm{ul}}, \\
& \operatorname{rank}\left(\overline{\mathbf{W}}_{\mathrm{E}}\right)=1, \\
& \overline{\mathbf{W}}_{\mathrm{E}} \succeq 0,
\end{aligned}
$$

where $\overline{\mathbf{W}}_{\mathrm{E}}=\overline{\mathbf{w}}_{\mathrm{E}} \overline{\mathbf{w}}_{\mathrm{E}}^{\dagger}, \overline{\mathbf{A}}_{k}=\left(\tilde{\mathbf{H}}_{\mathrm{au}, k}^{\dagger} \tilde{\mathbf{H}}_{\mathrm{au}, k} \otimes \mathbf{I}_{K_{\mathrm{ul}}}\right)$ and $\overline{\mathbf{B}}_{k}=\left(\mathbf{h}_{\mathrm{au}, k}^{\dagger} \mathbf{h}_{\mathrm{au}, k} \otimes \mathbf{I}_{K_{\mathrm{ul}}}\right)$, and we applied the SDR technique to relax the quadratic terms of the beamformers in the objective function and constraints. The optimization problem (34) is still non-convex (even w.r.t $\left\{\overline{\mathbf{W}}_{\mathrm{E}}, \tau_{k}\right\}$ ) due to non-convex constraints (34b) and (34e). In order to circumvent the non-convexity of the (34b), let us introduce $\bar{\tau}_{k}=\left(\tau_{k}\right)^{-1}$ and $f_{k}\left(\bar{\tau}_{k}\right)=\log _{2}\left(\bar{\tau}_{k}\right)$. Since $f_{k}\left(\bar{\tau}_{k}\right)$ is concave, we have

$$
f_{k}\left(\bar{\tau}_{k}\right) \leq f_{k}\left(\bar{\tau}_{k, 0}\right)+\frac{\partial f_{k}\left(\bar{\tau}_{k, 0}\right)}{\partial \bar{\tau}_{k}}\left(\bar{\tau}_{k}-\bar{\tau}_{k, 0}\right)
$$

Now by invoking (35), we approximate the objective function in (34) by its lower bound. Therefore, for a given $\alpha$, by dropping the rank-one constraint, problem (34) becomes

$$
\begin{aligned}
\max _{\overline{\mathbf{W}}_{\mathrm{E}}, \bar{\tau}_{k}} & \sum_{k=1}^{K_{\mathrm{dl}}} \log _{2}\left(\bar{\tau}_{k}+\Lambda^{i}(\alpha)\right)- \\
& \frac{1}{\log (2)} \sum_{k=1}^{K_{\mathrm{dl}}}\left(\log \left(\bar{\tau}_{k, 0}\right)+\frac{1}{\bar{\tau}_{k, 0}}\left(\bar{\tau}_{k}-\bar{\tau}_{k, 0}\right)\right), \\
\text { s.t } \quad & \frac{\eta \alpha}{(1-\alpha)} \operatorname{tr}\left(\overline{\mathbf{W}}_{\mathrm{E}} \overline{\mathbf{A}}_{k}\right)+\Phi_{k}^{i}(\alpha) \leqslant \bar{\tau}_{k}, \\
& \forall k \in\left\{1, \cdots, K_{\mathrm{dl}}\right\}, \\
& \sum_{k=1}^{K_{\mathrm{ul}}} \log _{2}\left(1+\Psi_{k}^{i}(\alpha) \operatorname{tr}\left(\overline{\mathbf{W}}_{\mathrm{E}} \overline{\mathbf{B}}_{k}\right)\right) \geq \frac{\bar{R}_{\mathrm{u}}}{(1-\alpha)}, \\
& \operatorname{tr}\left(\overline{\mathbf{W}}_{\mathrm{E}}\right)=K_{\mathrm{ul}}, \\
& \overline{\mathbf{W}}_{\mathrm{E}} \succeq 0 .
\end{aligned}
$$

The optimization (34) is then solved by solving (36) in conjunction with a line search over $\alpha$. The value of $\alpha$ and $\mathbf{W}_{E}$ that maximize the objective function in (34) are retrieved as optimal solutions. Unfortunately, it is not possible to guarantee that (36) always yields rank-one solutions. However, in our numerical simulations, we have found that (41) yields rank-one solutions with a very high probability. We outline the proposed optimization method in Algorithm 1.

Since (34) needs to be solved with successive convex approximation for each $\alpha$, it can be said that the complexity of Algorithm 1 is high, although exact computational complexity analysis is beyond the scope of this paper. In our future work, we will consider methods to decrease this complexity.

As an special case, we consider genie aided receiver case. By invoking (12) and after some algebraic manipulation, the downlink spectral efficiency with MRC/MRT and ZF processing can be approximated as 


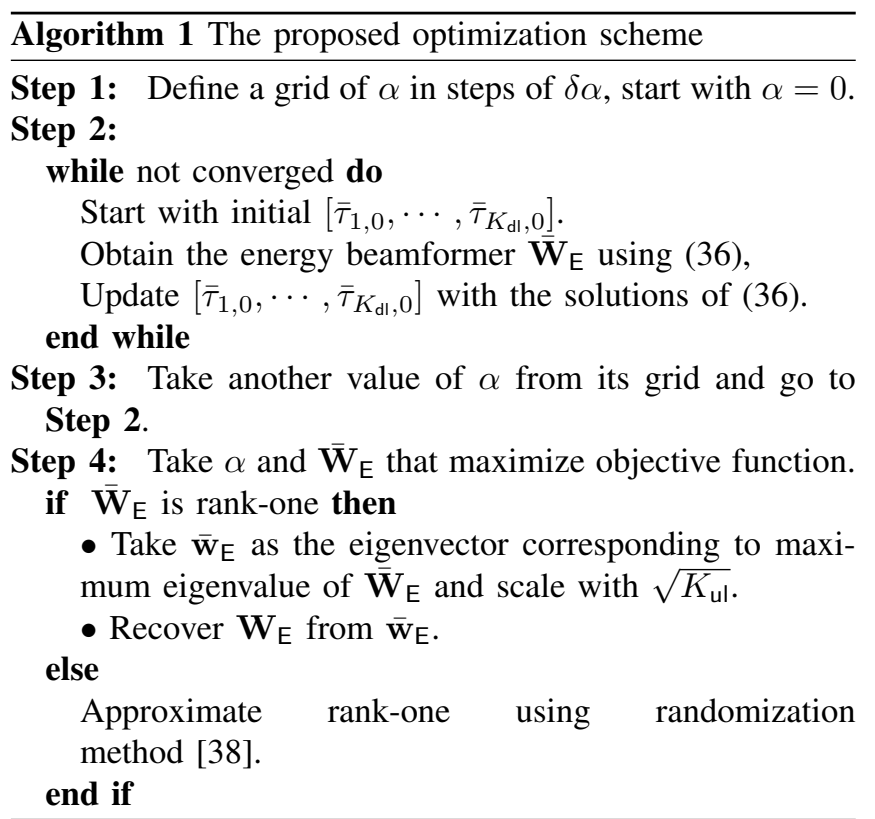

$$
\begin{aligned}
R_{\mathrm{D}}^{i}\left(\mathbf{W}_{\mathrm{E}}, \alpha\right) \approx & (1-\alpha) \sum_{k=1}^{K_{\mathrm{dl}}} \log _{2}(1+ \\
& \left.\frac{\Xi^{i}}{\frac{\eta \alpha}{1-\alpha} \operatorname{tr}\left(\tilde{\mathbf{H}}_{\mathrm{au}, k} \mathbf{W}_{\mathrm{E}} \mathbf{W}_{\mathrm{E}}^{\dagger} \tilde{\mathbf{H}}_{\mathrm{au}, k}^{\dagger}\right)+\Upsilon^{i}}\right),
\end{aligned}
$$

where $i \in\{\mathrm{MR}, \mathrm{ZF}\}, \Xi^{\mathrm{MR}}=\rho_{\mathrm{a}} \alpha_{\mathrm{MRT}}^{2}\left\|\mathrm{~g}_{\mathrm{ad}, k}\right\|^{4}, \Upsilon^{\mathrm{MR}}=$

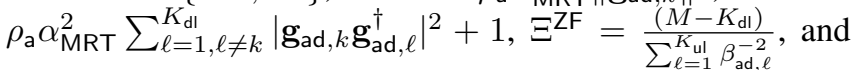
$\Upsilon^{\mathrm{ZF}}=1 / \rho_{\mathrm{a}}$.

Furthermore, the uplink spectral efficiency of the genie aided HAP with MRC/MRT and ZF processing can be expressed as

$$
\begin{aligned}
& R_{\mathrm{U}}^{\mathrm{MR}}\left(\mathbf{W}_{\mathrm{E}}, \alpha\right)=(1-\alpha) \sum_{k=1}^{K_{\mathrm{ul}}} \log _{2}(1+ \\
& \left.\frac{\kappa \beta_{\mathrm{au}, k} \operatorname{tr}\left(\mathbf{h}_{\mathrm{au}, k}^{\dagger} \mathbf{h}_{\mathrm{au}, k} \mathbf{W}_{\mathrm{E}} \mathbf{W}_{\mathrm{E}}^{\dagger}\right)\left\|\mathbf{g}_{\mathrm{ua}, k}\right\|^{2}}{\kappa \sum_{\ell \neq k}^{K_{\mathrm{ul}}} \beta_{\mathrm{au}, \ell}^{2}\left|\hat{g}_{\ell}\right|^{2} \operatorname{tr}\left(\mathbf{h}_{\mathrm{au}, \ell}^{\dagger} \mathbf{h}_{\mathrm{au}, \ell} \mathbf{W}_{\mathrm{E}} \mathbf{W}_{\mathrm{E}}^{\dagger}\right)+k_{1} \sigma_{\mathrm{SI}}^{2}+1 / \rho_{\mathrm{a}}}\right),
\end{aligned}
$$

and

$$
\begin{aligned}
R_{\mathrm{U}}^{\mathrm{ZF}}\left(\mathbf{W}_{\mathrm{E}}, \alpha\right)=(1-\alpha) \sum_{k=1}^{K_{\mathrm{ul}}} \log _{2}(1+ \\
\left.\frac{\kappa \beta_{\mathrm{au}, k}\left(N-K_{\mathrm{ul}}\right) \operatorname{tr}\left(\mathbf{h}_{\mathrm{au}, k}^{\dagger} \mathbf{h}_{\mathrm{au}, k} \mathbf{W}_{\mathrm{E}} \mathbf{W}_{\mathrm{E}}^{\dagger}\right)}{k_{1} \sigma_{\mathrm{Sl}}^{2}+1 / \rho_{\mathrm{a}}}\right),
\end{aligned}
$$

respectively. Note that with $R_{\mathrm{U}}^{\mathrm{MR}}\left(\mathbf{W}_{\mathrm{E}}, \alpha\right)$ in (38), the optimization problem (26) is not tractable (or convex). Fortunately, when $N$ is large, by using the law of large numbers, we can neglect the intra-sensor interference. In the numerical results, we show that this approximation is very tight and fairly accurate. Therefore, by neglecting the intra-sensor interference term in (38), we obtain

$$
R_{\mathrm{U}}^{i}\left(\mathbf{W}_{\mathrm{E}}, \alpha\right)=(1-\alpha) \sum_{k=1}^{K_{\mathrm{ul}}} \log _{2}(1+
$$

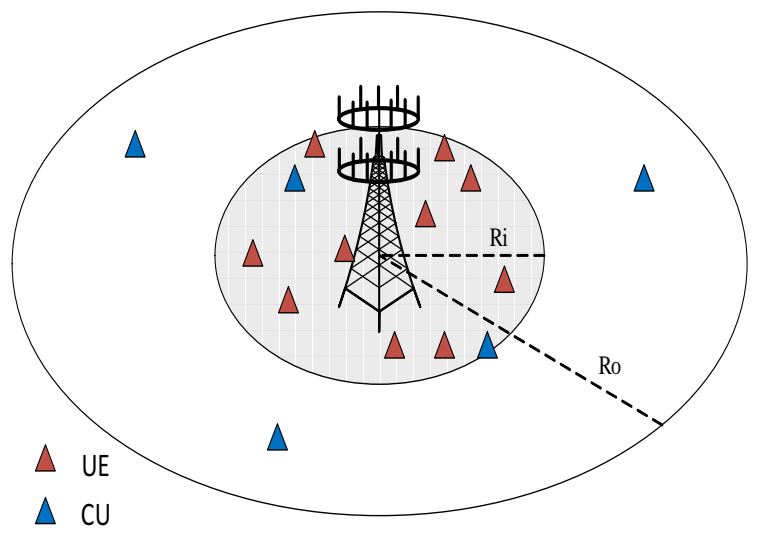

Fig. 2: A system topology with $K_{\mathrm{dl}}$ CUs and $K_{\mathrm{ul}}$ UEs located within a circle of $250 \mathrm{~m}$ radius is used in numerical examples.

$$
\left.\frac{\alpha \Theta^{i}}{(1-\alpha)} \operatorname{tr}\left(\mathbf{h}_{\mathrm{au}, k}^{\dagger} \mathbf{h}_{\mathrm{au}, k} \mathbf{W}_{\mathrm{E}} \mathbf{W}_{\mathrm{E}}^{\dagger}\right)\right),
$$

where $\Theta^{\mathrm{MR}}=\frac{\eta \beta_{\mathrm{au}, k}\left\|\mathbf{g}_{\mathrm{ua}, k}\right\|^{2}}{k_{1} \sigma_{\mathrm{SI}}^{2}+1 / \rho_{\mathrm{a}}}$ and $\Theta^{\mathrm{ZF}}=\frac{\eta \beta_{\mathrm{au}, k}\left(N-K_{\mathrm{ul}}\right)}{k_{1} \sigma_{\mathrm{SI}}^{2}+1 / \rho_{\mathrm{a}}}$.

To this end, when $N$ grows large, the optimization problem (27) can be expressed as

$$
\begin{aligned}
\max _{\mathbf{W}_{\mathrm{E}}, 0 \leq \alpha \leq 1}(1-\alpha) \sum_{k=1}^{K_{\mathrm{dl}}} \log _{2}(1+ & \left.\frac{\Xi^{i}}{\frac{\eta \alpha}{(1-\alpha)} \operatorname{tr}\left(\tilde{\mathbf{H}}_{\mathrm{au}, k} \mathbf{W}_{\mathrm{E}} \mathbf{W}_{\mathrm{E}}^{\dagger} \tilde{\mathbf{H}}_{\mathrm{au}, k}^{\dagger}\right)+\Upsilon^{i}}\right), \\
\text { s.t } \quad & (1-\alpha) \sum_{k=1}^{K_{\mathrm{ul}}} \log _{2}(1+ \\
& \left.\frac{\alpha \Theta^{i}}{(1-\alpha)} \operatorname{tr}\left(\mathbf{h}_{\mathrm{au}, k}^{\dagger} \mathbf{h}_{\mathrm{au}, k} \mathbf{W}_{\mathrm{E}} \mathbf{W}_{\mathrm{E}}^{\dagger}\right)\right) \geq \bar{R}_{\mathrm{u}}, \\
& \left\|\mathbf{w}_{\mathrm{E}, k}\right\|=1, \quad k=1, \cdots, K_{\mathrm{ul}} .
\end{aligned}
$$

For a given $\alpha$, the optimization problem (41) can be solved in a similar way as that for problem (31). In particular, we successively approximate (41) as an SDR to optimize $\mathbf{W}_{\mathrm{E}}$, in conjunction with a line search over $\alpha$.

\section{NumericAl RESUlts AND DisCUSSION}

In this section, simulation results are presented to verify the effectiveness of the proposed beamforming scheme. We consider a wireless powered massive network as shown in Fig. 2, where cell radius is considered to be $R_{o}=250 \mathrm{~m}$. The HAP is located at the center of cell, $K_{\mathrm{ul}}=5$ UEs are randomly located within a reference distance (RD) of $R_{i}=25 \mathrm{~m}$ from the HAP, which is the case for smallcell scenarios [21], [24], and $K_{\mathrm{dl}}=3$ CUs are randomly distributed across the whole of the cell. we assume that $p_{p}=$ $p_{\mathrm{a}}$ and the energy conversion efficiency is set to be $\eta=0.7$.

As for simulation parameters, we use the HataCOST231 and 3-slope path loss model [39] to imitate the large-scale 
TABLE I: System Parameters for the Simulations.

\begin{tabular}{|l|l|}
\hline Parameter & Value \\
\hline Carrier frequency & $1900 \mathrm{MHz}$ \\
\hline Bandwidth $(B)$ & $20 \mathrm{MHz}$ \\
\hline$\sigma_{s h}$ & $8 \mathrm{~dB}$ \\
\hline Noise Figure $(F)$ & $9 \mathrm{~dB}$ \\
\hline HAP antenna height & $20 \mathrm{~m}$ \\
\hline CU and UE antenna height & $1.5 \mathrm{~m}$ \\
\hline$d_{1}, d_{0}$ & $50,10 \mathrm{~m}$ \\
\hline
\end{tabular}

fading coefficients, i.e. $\beta_{\mathrm{au}, k}=10^{\left(\mathcal{S}+P L\left(d_{\mathrm{au} k}\right)\right) / 10}, \beta_{\mathrm{ad}, k}=$ $10^{\left(\mathcal{S}+P L\left(d_{\mathrm{ad} k}\right)\right) / 10}$, and $\beta_{\mathrm{ud}, k \ell}=10^{\left(\mathcal{S}+P L\left(d_{\mathrm{ud} k \ell}\right)\right) / 10}$,

where $\mathcal{S} \sim \mathcal{N}\left(0, \sigma_{s h}^{2}\right)$ presents the shadowing fading effect and

$P L(d)=\left\{\begin{array}{lc}-L-35 \log _{10}(d) & d>d_{1} \\ -L-15 \log _{10}\left(d_{1}\right)-20 \log _{10}(d) & d_{0}<d \leq d_{1}, \\ -L-15 \log _{10}\left(d_{1}\right)-20 \log _{10}\left(d_{0}\right) & d \leq d_{0}\end{array}\right.$

with $d \equiv d_{\mathrm{au} k}\left(d \equiv d_{\mathrm{ad} k}, d \equiv d_{\mathrm{ud} k \ell}\right)$ being the distance in $\mathrm{km}$ between the HAP and UE $k$ (between the HAP and CU $k$, between $\mathrm{UE} k$ and $\mathrm{CU} \ell$ ) and

$$
\begin{aligned}
L & =46.3+33.9 \log _{10}(f)-13.92 \log _{10}\left(h_{\mathrm{a}}\right) \\
& -\left(1.1 \log _{10}(f)-0.7\right) h_{\mathrm{u}}+\left(1.56 \log _{10}(f)-0.8\right),
\end{aligned}
$$

where $f$ is the carrier frequency (in $\mathrm{MHz}$ ), $h_{\mathrm{a}}$ is the HAP antenna height (in $\mathrm{m}$ ), and $h_{\mathrm{u}}$ denotes the user antenna height (in $\mathrm{m}$ ). The large-scale fading coefficient of the SI link at the $\mathrm{HAP}$ is calculated as $\sigma_{\mathrm{SI}}^{2}=10^{\frac{P L_{\mathrm{SI}}}{10}}$ where $P L_{\mathrm{SI}}=-81 \mathrm{~dB}$. Unless specifically stated otherwise, similar to [39], other parameters are given in Table I. Therefore, noise power $\sigma_{n}^{2}=$ $k_{B} T_{0} B F=-92 \mathrm{dBm}$, where $k_{B}=1.381 \times 10^{-23}$ Joules $/ \mathrm{K}$ is the Boltzmann constant and $T_{0}=290 \mathrm{~K}$ is the noise temperature.

Fig. 3 shows the achievable downlink and uplink sumrate of the system with MRC/MRT and ZF processing. The "Simulation" curves represent the sum-rates obtained from the outputs of a Monte-Carlo simulator using (13), while the "Analytical" curves represent the achievable sum-rates obtained by using Propositions 2- 5. It can be seen that the derived lower bounds are tight for a large range of transmit power, specially in case of ZF processing. This implies that our analytical closed-form results form a good predictor of the system performance. Moreover, from Fig. 3(a), it can be observed that at low transmit power levels, where the intra-sensor interference is not significant, MRC/MRT outperforms the $\mathrm{ZF}$ and vice versa at high transmit power levels. Furthermore, the achievable uplink sum-rate obtained from both MRC/MRT and ZF processing are saturated in the high transmit power region due to imperfect SI cancellation at the HAP.

Fig. 4 shows the achievable sum-rate of the system with the MRT/MRC and ZF processing for different number of transmit and receive antennas at the HAP. We can see that the proposed approximations and lower bounds are very

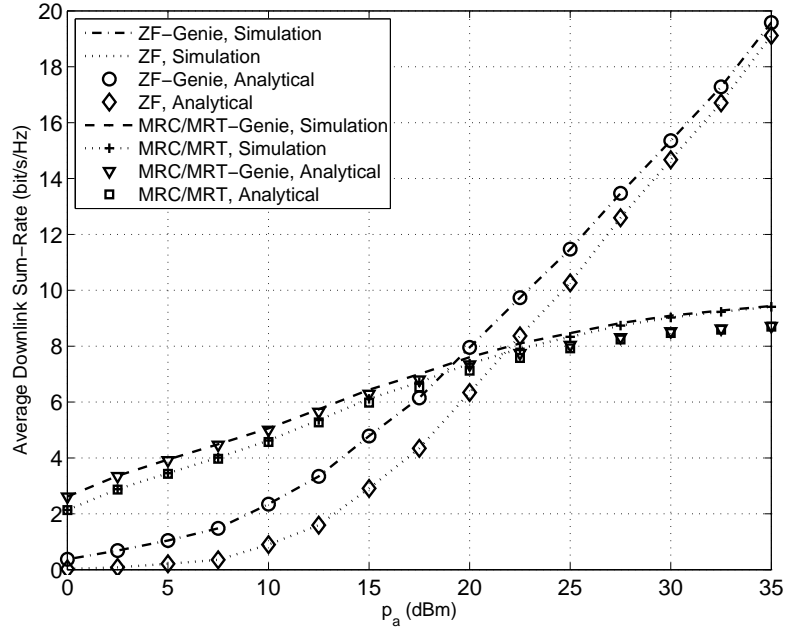

(a) Downlink

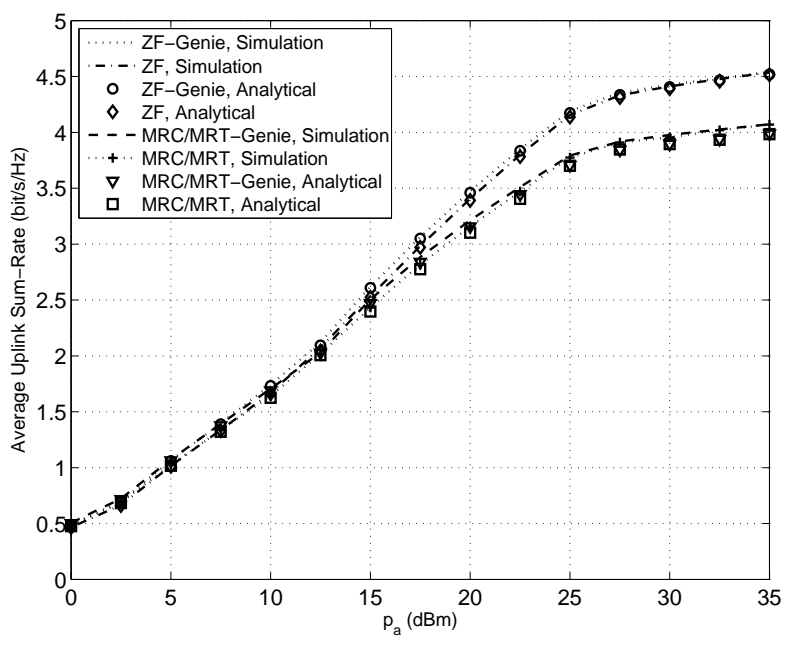

(b) Uplink

Fig. 3: Average sum-rates versus $p_{\mathrm{a}}(M=N=100, \alpha=$ $\left.0.5, k_{1}=0.01\right)$.

tight, especially for large antenna array. or a transmitter with a large number of antennas, $M, \mathrm{ZF}$ processing provides a constant gain over the MRC/MRT processing, which confirms our finding in Remark 3. Furthermore, it can be observed from Fig. 4(b) that the difference in the achievable uplink sum-rate between ZF and MRC/MRT processing is significant when the number of antennas is large. This is because in the MRC/MRT processing the intr-pair interference term in (17) scales with number of receive antennas, $N$, while in the ZF processing the intra-pair interference is completely cancelled out.

Fig. 5 illustrates the impact of the number of transmit (receive) antennas at the HAP on the achievable sum-rate of the proposed MRC/MRT and ZF processing schemes. It is noted that average uplink (downlink) sum-rate of the system remains almost constant when increasing the number of transmit (receive) antennas for $N=100(M=100)$. This observation is important as it provides the possibility of deploying large antenna-array just at the transmit (receive) 


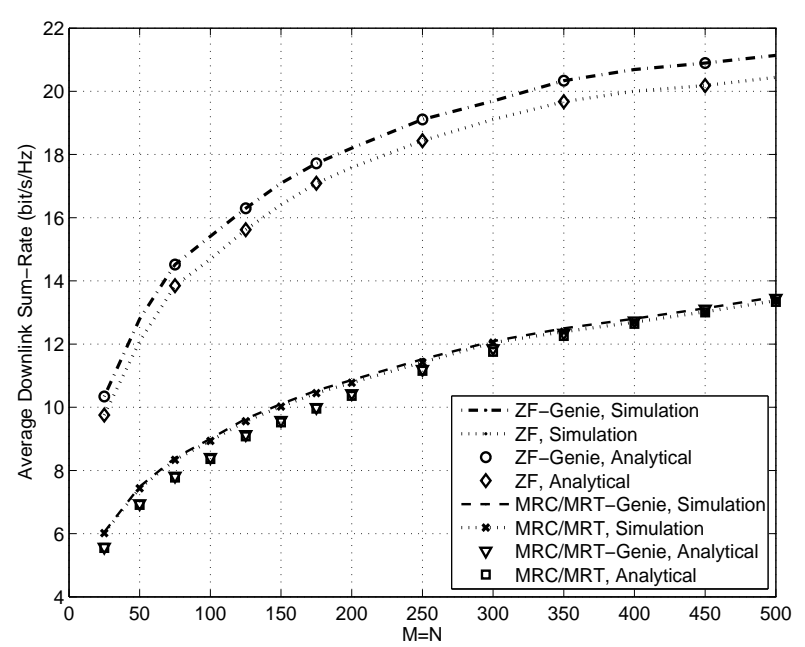

(a) Downlink

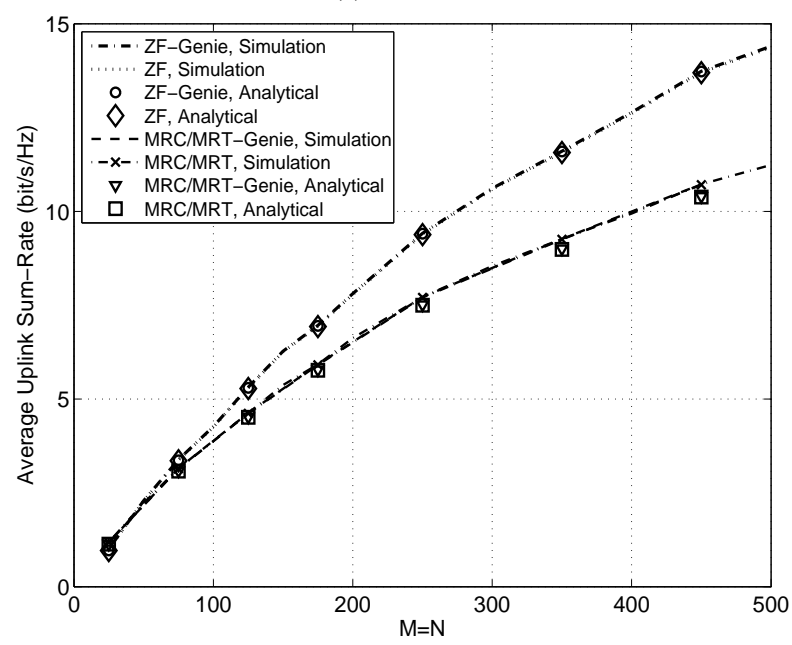

(b) Uplink

Fig. 4: Average sum-rates versus the number of transmit (receive) antennas the $\operatorname{HAP}\left(p_{\mathrm{a}}=30 \mathrm{dBm}, \alpha=0.5, k_{1}=\right.$ $0.01)$.

side of the HAP, when a predefined achievable rate (or a QoS requirement) is desired at the uplink (downlink).

Fig. 6 shows the rate regions obtained with the proposed Algorithm 1 with MRC/MRT and ZF processing. We further included the rate region of a low-complexity energy beamforming design as a benchmark, at which a ZF constraint has been considered to ensures no interference at the UEs from the CUs. In this case, the optimization problem is

$$
\begin{array}{cl}
\max _{\left\|\overline{\mathbf{w}}_{\mathrm{E}}\right\|^{2}=K_{\mathrm{ul}}} & \overline{\mathbf{w}}_{\mathrm{E}}^{\dagger} \overline{\mathbf{B}} \overline{\mathbf{w}}_{\mathrm{E}}, \\
\text { s.t } & \overline{\mathbf{w}}_{\mathrm{E}}^{\dagger} \mathbf{A}_{k} \overline{\mathbf{w}}_{\mathrm{E}}=0, \forall k \in\left\{1, \cdots, K_{\mathrm{dl}}\right\}
\end{array}
$$

To solve the problem in (44), we first apply SDR technique by using a positive semidefinite matrix $\overline{\mathbf{W}}_{\mathrm{E}}=\overline{\mathbf{w}}_{\mathrm{E}} \overline{\mathbf{w}}_{\mathrm{E}}^{\dagger}$ and relaxing the rank-constraint on $\overline{\mathbf{W}}_{\mathrm{E}}$. The relaxed optimization problem (44) in terms of $\overline{\mathbf{W}}_{\mathrm{E}}$ is

$$
\begin{array}{ll}
\max _{\mathbf{W}_{\mathrm{E}}} & \operatorname{tr}\left(\overline{\mathbf{W}}_{\mathrm{E}} \overline{\mathbf{B}}\right), \\
\text { s.t } & \operatorname{tr}\left(\overline{\mathbf{W}}_{\mathrm{E}} \mathbf{A}_{k}\right)=0, \quad \forall k \in\left\{1, \cdots, K_{\mathrm{dl}}\right\},
\end{array}
$$

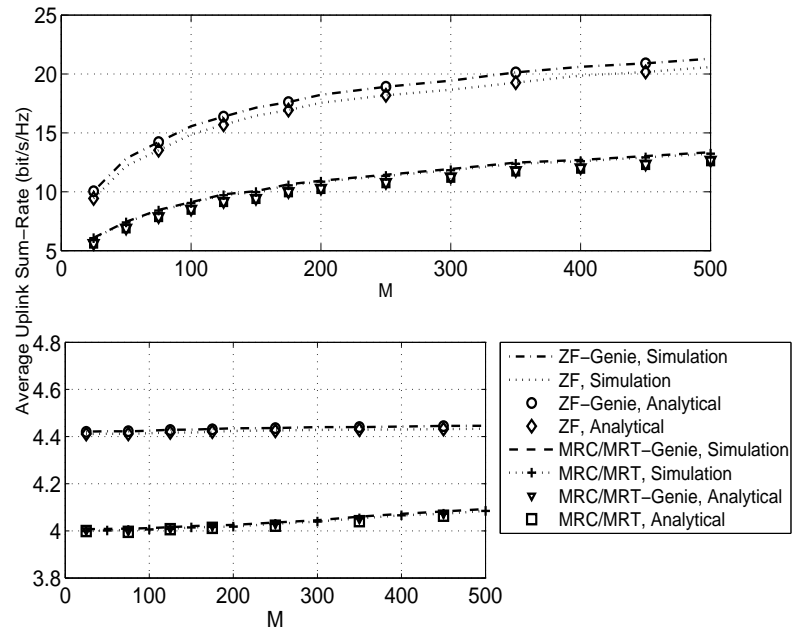

(a) $N=100$
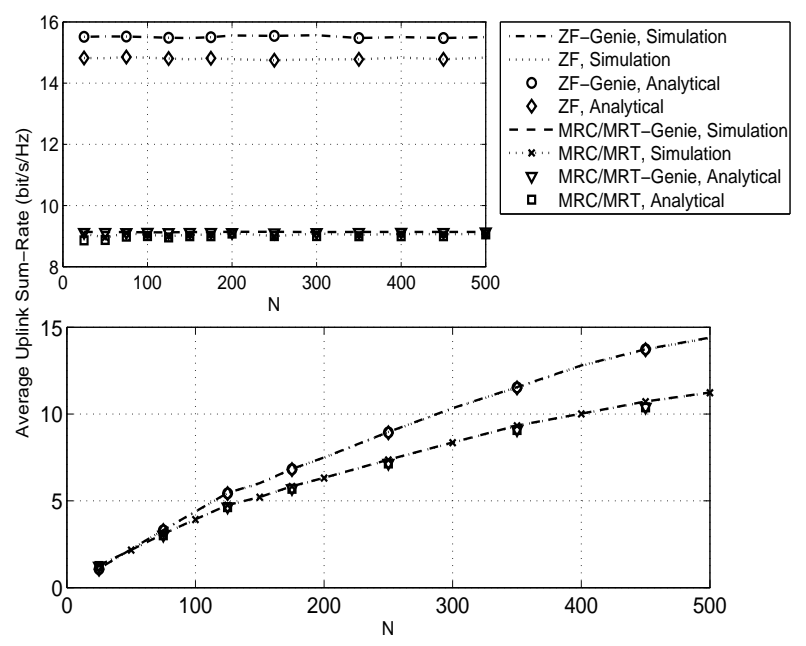

(b) $M=100$

Fig. 5: Average sum-rates versus the number of transmit (receive) antennas and for a fixed number of receive (transmit) antennas at the $\operatorname{HAP}\left(p_{\mathrm{a}}=30 \mathrm{dBm}, \alpha=0.5, k_{1}=0.01\right)$.

$$
\operatorname{tr}\left(\overline{\mathbf{W}}_{\mathrm{E}}\right)=K_{\mathrm{ul}}, \quad \overline{\mathbf{W}}_{\mathrm{E}} \succeq 0 .
$$

The optimization problem (45) is a standard SDR problem. Let $\overline{\mathbf{W}}_{\mathrm{E}}^{*}$ be the optimum solution of (45). The beamforming vector $\overline{\mathbf{w}}_{\mathrm{E}}^{*}$ is obtained $\overline{\mathbf{w}}_{\mathrm{E}}^{*}=\sqrt{K_{\mathrm{ul}}} \tilde{\mathbf{u}} \tilde{\mathbf{u}}^{\dagger}$, where $\tilde{\mathbf{u}}$ is the eigenvector corresponding to non-zero eigenvalue of $\overline{\mathbf{W}}_{\mathrm{E}}^{*}$. Accordingly $\mathbf{W}_{\mathrm{E}}$ can be extracted from $\tilde{\mathbf{w}}_{\mathrm{E}}$ and then $\alpha$ is numerically obtained from (31). As expected, the proposed Algorithm 1 significantly outperforms the benchmark schemes. This observation can be explained as follows. The energy beamformers used in the benchmark scheme try to cancel the interference inflicted on the UEs from CUs and maximize the achievable uplink rate. However, it does not take it to consideration that UEs can transmit their uplink data in the following slot with high powers if they harvest more energy in the energy harvesting phase. As such, UE nodes can produce significant interference to downlink data transmission from HAP. On the other hand, the proposed energy beamforming and harvesting time design and tries to 


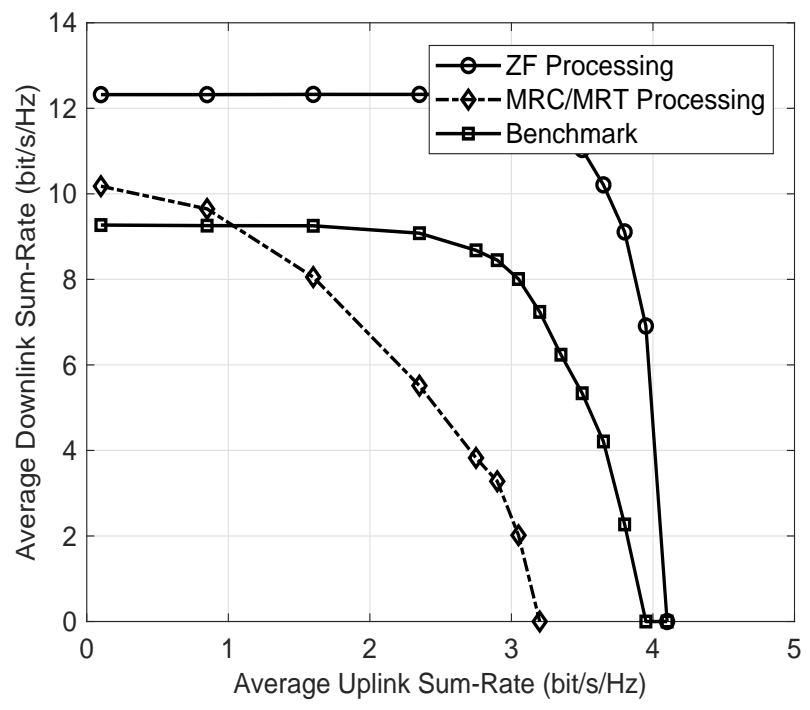

Fig. 6: Average downlink rate versus average uplink rate for the proposed energy beamforming design $(N=M=50$, $\left.p_{\mathrm{a}}=30 \mathrm{dBm}\right)$.

maintain a tradeoff between energy transfer to UEs and the interference they generate, due to the harvested energy, to downlink transmission from HAP.

\section{CONCLUSION}

We have characterized the achievable uplink and downlink rate of a massive MIMO-enabled information and energy transfer system with the time-switching protocol. In particular, we have derived the approximate and asymptotic achievable rate expressions, which provide an efficient means to evaluate the impact of the number of antennas, number of uplink and downlink users, and channel estimate errors on the achievable uplink and downlink rate of the considered system. We further quantified the performance gain achieved by using ZF processing at the HAP over the MRC/MRT processing. We found that for large transmit antenna arrays, $\mathrm{ZF}$ processing provides a constant downlink gain over the MRC/MRT processing that depends on the number of downlink users and their channel estimation accuracy. In order to maintain a tradeoff between energy transfer to UEs and the interference they generate to downlink transmission from HAP, due to the harvested energy, we have proposed a joint energy beamforming and harvesting time design at the HAP. Specifically, downlink sum-rate of CUs has been maximized, while ensuring that the uplink sum-rate of UEs is above a certain threshold. The proposed energy beamforming design ensures a more balanced rate region between the uplink and downlink as compared with the suboptimum energy beamformer design.

\section{APPENDIX A}

PROOF OF PROPOSITION 1

By substituting $\mathbf{w}_{r, k}^{\mathrm{MRC}}, p_{\mathrm{u}, k}$ and $p_{\mathrm{u}, \ell}$ into (9), the achievable uplink rate of the $k$-th UE can be expressed as (46) at the top of the next page, where $\hat{g}_{\ell}=\frac{\mathbf{g}_{\text {uа }, k}^{\dagger} \mathbf{g}_{\text {иа }, \ell}}{\left\|\mathbf{g}_{\text {uа }, k}\right\|}$. When $N$ grows large, we have

$$
\begin{aligned}
\sum_{\substack{j=1 \\
j \neq \ell}}^{K_{\mathrm{ul}}} \frac{\left|\mathbf{g}_{\mathrm{au}, k} \mathbf{g}_{\mathrm{au}, j}^{\dagger}\right|^{2}}{\left\|\mathbf{g}_{\mathrm{au}, j}\right\|^{2}}=\mathbf{g}_{\mathrm{au}, k}\left(\sum_{\substack{j=1 \\
j \neq k}}^{K_{\mathrm{ul}}} \frac{\mathbf{g}_{\mathrm{au}, j}^{\dagger} \mathbf{g}_{\mathrm{au}, j}}{\left\|\mathbf{g}_{\mathrm{au}, j}\right\|^{2}}\right) \mathbf{g}_{\mathrm{au}, k}^{\dagger} \\
\stackrel{N \rightarrow \infty}{\longrightarrow} \beta_{\mathrm{au}, k} \operatorname{tr}(\mathbf{G})=\beta_{\mathrm{au}, k}\left(K_{\mathrm{ul}}-1\right),
\end{aligned}
$$

where $\mathbf{G}=\sum_{\substack{j=1 \\ j \neq k}}^{K_{\mathrm{ul}}} \frac{\mathbf{g}_{\mathrm{au}, j}^{\dagger} \mathbf{g}_{\mathrm{au}, j}}{\left\|\mathbf{g}_{\mathrm{au}, j}\right\|^{2}}$. Therefore, the achievable uplink rate in (46) can be written as (48) at the top of the next page. Now, we focus on the SI term in (48). Let us denote $Z_{k}=\mathrm{E}_{\tilde{\mathcal{E}}_{\mathrm{SI}}}\left\{\left|\mathbf{w}_{r, k}^{\dagger} \tilde{\mathcal{E}}_{\mathrm{SI}}^{T} \hat{\mathbf{w}}_{t, \ell}\right|^{2} \mid \mathbf{w}_{r, k}, \hat{\mathbf{w}}_{t, \ell}\right\}$. Conditioned on $\mathbf{w}_{r, k}, \mathbf{w}_{r, k}^{\dagger} \tilde{\mathcal{E}}_{\mathrm{SI}}^{T}$ is a Gaussian random vector with zero mean and covariance matrix

$$
\begin{aligned}
\boldsymbol{\Omega}_{Z} & \stackrel{(a)}{=}\left\|\mathbf{w}_{r, k}\right\|^{2}\left(k_{1} \sigma_{\mathrm{SI}}^{2}+\sigma_{\mathrm{SI}}^{2}-\sigma_{\mathrm{SI}, k}^{2}\right) \mathbf{I}_{M} \\
& \stackrel{(b)}{=}\left\|\mathbf{w}_{r, k}\right\|^{2}\left(k_{1} \sigma_{\mathrm{SI}}^{2}+\frac{1}{1+\tau p_{\mathrm{a}} \sigma_{\mathrm{SI}}^{2}}\right) \mathbf{I}_{M} .
\end{aligned}
$$

where (a) is obtained by using the fact that the rows of $\mathcal{E}_{\mathrm{SI}}$ are distributed as $\mathcal{C} \mathcal{N}\left(\mathbf{0}, \sigma_{\mathrm{SI}}^{2} \mathbf{I}_{\mathbf{N}}-\boldsymbol{\Omega}_{\mathrm{SI}}\right)$ and (b) is obtained by using $\sigma_{\mathrm{SI}, k}^{2}$. Since the covariance matrix does not depend on $\mathbf{w}_{r, k}, \mathbf{w}_{r, k}^{\dagger} \tilde{\mathcal{E}}_{\mathrm{SI}}^{T}$ is a Gaussian distributed vector and independent of $\mathbf{w}_{r, k}$. Therefore, $Z_{k}=\mathrm{E}_{\tilde{\mathcal{E}}_{\mathrm{SI}}}\left\{\left|\overline{\mathcal{E}}_{\mathrm{SI}} \hat{\mathbf{w}}_{t, \ell}\right|^{2} \mid \hat{\mathbf{w}}_{t, \ell}\right\}$ where $\overline{\mathcal{E}}_{\mathrm{SI}}=\mathbf{w}_{r, k}^{\dagger} \tilde{\mathcal{E}}_{\mathrm{SI}}^{T}$. Accordingly, we can obtain

$$
\begin{aligned}
Z_{k} & =\mathrm{E}_{\overline{\mathcal{E}}_{\mathrm{SI}}}\left\{\hat{\mathbf{w}}_{t, \ell}^{\dagger} \overline{\mathcal{E}}_{\mathrm{SI}} \overline{\mathcal{E}}_{\mathrm{SI}} \hat{\mathbf{w}}_{t, \ell} \mid \hat{\mathbf{w}}_{t, \ell}\right\} \\
& =\left(k_{1} \sigma_{\mathrm{SI}}^{2}+\frac{1}{1+\tau p_{\mathrm{a}} \sigma_{\mathrm{SI}}^{2}}\right)\left\|\mathbf{w}_{r, k}\right\|^{2}\left\|\hat{\mathbf{w}}_{t, \ell}\right\|^{2} .
\end{aligned}
$$

Hence, by substituting (49) into (48) and recalling that $\alpha_{\mathrm{MRT}}^{2}=\frac{1}{M \sum_{\ell=1}^{K_{\mathrm{dl}}} \sigma_{\mathrm{ad}, \ell}^{2}}$, the achievable uplink rate (46) can be approximated as (50) at the top of the next page.

We notice that $\left\|\mathbf{g}_{\mathrm{au}, k}\right\|^{2}$ follows Chi-square distribution with $2 N$ degrees-of-freedom. Moreover, conditioned on $\mathrm{g}_{\mathrm{au}, k}, \hat{g}_{\ell}$ is zero-mean Gaussian RV with variance $\beta_{\mathrm{au}, \ell}$, which does not depend on $\mathbf{g}_{\mathrm{au}, k}$. Therefore, $\hat{g}_{\ell}$ is Gaussian RV and independent of $\mathbf{g}_{\mathrm{au}, k}, \hat{g}_{\ell} \sim \mathcal{C N}\left(0, \beta_{\mathrm{au}, \ell}\right)$. Accordingly, using the moment generating function (MGF) approach [40, Lemma 1], uplink achievable rate can be obtained as (16).

\section{APPENDIX B ProOF OF PROPOSITION 2}

From (50), by the convexity of $\log _{2}\left(1+\frac{1}{x}\right)$ and using Jensen's inequality, the lower bound on the achievable rate is obtained as (51) at the top of the next page.

Let $\omega \triangleq p_{\mathrm{a}}\left(k_{1} \sigma_{\mathrm{SI}}^{2}+\frac{\sigma_{\mathrm{SI}}^{2}}{1+\tau p_{\mathrm{a}} \sigma_{\mathrm{SI}}^{2}}\right)+\sigma_{n}^{2}$. Since $\hat{g}_{\ell}$ is independent of $\mathbf{g}_{\mathrm{au}, k}$, we have

$$
\mathrm{E}\left\{\frac{\kappa p_{\mathrm{a}}\left(K_{\mathrm{ul}}+N-1\right) \sum_{\ell \neq k}^{K_{\mathrm{ul}}} \beta_{\mathrm{au}, \ell}\left|\hat{g}_{\ell}\right|^{2}+\omega}{\kappa p_{\mathrm{a}} \beta_{\mathrm{au}, k}\left(K_{\mathrm{ul}}+N-1\right)\left\|\mathbf{g}_{\mathrm{au}, k}\right\|^{2}}\right\}
$$




$$
\begin{aligned}
& R_{\mathrm{ul}, \mathrm{k}}^{\mathrm{MR}}=(1-\alpha) \mathrm{E}\left\{\log _{2}(1+\right. \\
& \left.\left.\frac{\kappa p_{\mathrm{a}}\left(\sum_{\substack{j=1 \\
j \neq k}}^{K_{\mathrm{ul}}} \frac{\left|\mathbf{g}_{\mathrm{au}, k} \mathbf{g}_{\mathrm{au}, j}^{\dagger}\right|^{2}}{\left\|\mathbf{g}_{\mathrm{au}, j}\right\|^{2}}+\left\|\mathbf{g}_{\mathrm{au}, k}\right\|^{2}\right)\left\|\mathbf{g}_{\mathrm{ua}, k}\right\|^{2}}{\kappa p_{\mathrm{a}} \sum_{\substack{\ell=1 \\
\ell \neq k}}^{K_{\mathrm{ul}}}\left(\sum_{\substack{j=1 \\
j \neq \ell}}^{K_{\mathrm{ul}}} \frac{\left|\mathbf{g}_{\mathrm{au}}, \ell \mathbf{g}_{\mathrm{au}, j}^{\dagger}\right|^{2}}{\left\|\mathbf{g}_{\mathrm{au}, j}\right\|^{2}}+\left\|\mathbf{g}_{\mathrm{au}, \ell}\right\|^{2}\right)\left|\hat{g}_{\ell}\right|^{2}+\frac{p_{\mathrm{a}}}{\alpha_{\mathrm{MRC}}^{2}\left\|\mathbf{g}_{\mathrm{ua}, k}\right\|^{2}} \sum_{\ell=1}^{K_{\mathrm{dll}}} \mathrm{E}_{\tilde{\mathcal{E}}_{\mathrm{sl}}}\left\{\left|\mathbf{w}_{r, k}^{\dagger} \tilde{\mathcal{E}}_{\mathrm{SI}}^{T} \hat{\mathbf{w}}_{t, \ell}\right|^{2}\right\}+\sigma_{n}^{2}}\right)\right\}, \\
& R_{\mathrm{ul}, \mathrm{k}}^{\mathrm{MR}}=(1-\alpha) \mathrm{E}\left\{\log _{2}(1+\right. \\
& \left.\left.\frac{\kappa p_{\mathrm{a}} \beta_{\mathrm{au}, k}\left(K_{\mathrm{ul}}+N-1\right)\left\|\mathbf{g}_{\mathrm{ua}, k}\right\|^{2}}{\kappa p_{\mathrm{a}}\left(K_{\mathrm{ul}}+N-1\right) \sum_{\substack{\ell=1 \\
\ell \neq k}}^{K_{\mathrm{ul}}} \beta_{\mathrm{au}, \ell}\left|\hat{g}_{\ell}\right|^{2}+\frac{p_{\mathrm{a}}}{\alpha_{\mathrm{MRC}}^{2}\left\|\mathrm{~g}_{\mathrm{ua}, k}\right\|^{2}} \sum_{\ell=1}^{K_{\mathrm{dl}}} \mathrm{E}_{\tilde{\mathcal{E}}_{\mathrm{sl}}}\left\{\left|\mathbf{w}_{r, k}^{\dagger} \tilde{\mathcal{E}}_{\mathrm{SI}}^{T} \hat{\mathbf{w}}_{t, \ell}\right|^{2}\right\}+\sigma_{n}^{2}}\right)\right\} . \\
& R_{\mathrm{ul}, \mathrm{k}}^{\mathrm{MR}} \approx(1-\alpha) \mathrm{E}\left\{\log _{2}\left(1+\frac{\kappa p_{\mathrm{a}} \beta_{\mathrm{au}, k}\left(K_{\mathrm{ul}}+N-1\right)\left\|\mathbf{g}_{\mathrm{au}, k}\right\|^{2}}{\kappa p_{\mathrm{a}}\left(K_{\mathrm{ul}}+N-1\right) \sum_{\ell \neq k}^{K_{\mathrm{ul}}} \beta_{\mathrm{au}, \ell}\left|\hat{g}_{\ell}\right|^{2}+p_{\mathrm{a}}\left(k_{1} \sigma_{\mathrm{Sl}}^{2}+\frac{1}{1+\tau p_{\mathrm{a}} \sigma_{\mathrm{sl}}^{2}}\right)+\sigma_{n}^{2}}\right)\right\} . \\
& R_{\mathrm{ul}, \mathrm{k}}^{\mathrm{MR}} \geq \tilde{R}_{\mathrm{ul}, \mathrm{k}}^{\mathrm{MR}}=(1-\alpha) \log _{2}\left(1+\left(\mathrm{E}\left\{\frac{\kappa p_{\mathrm{a}}\left(K_{\mathrm{ul}}+N-1\right) \sum_{\ell \neq k}^{K_{\mathrm{ul}}} \beta_{\mathrm{au}, \ell}\left|\hat{g}_{\ell}\right|^{2}+p_{\mathrm{a}}\left(k_{1} \sigma_{\mathrm{Sl}}^{2}+\frac{\sigma_{\mathrm{Sl}}^{2}}{1+\tau p_{\mathrm{a}} \sigma_{\mathrm{Sl}}^{2}}\right)+\sigma_{n}^{2}}{\kappa p_{\mathrm{a}} \beta_{\mathrm{au}, k}\left(K_{\mathrm{ul}}+N-1\right)\left\|\mathbf{g}_{\mathrm{au}, k}\right\|^{2}}\right\}\right)^{-1}\right) .
\end{aligned}
$$

$$
\begin{aligned}
& =\left(\kappa p_{\mathrm{a}}\left(K_{\mathrm{ul}}+N-1\right) \sum_{\ell \neq k}^{K_{\mathrm{ul}}} \beta_{\mathrm{au}, \ell} \mathrm{E}\left\{\left|\hat{g}_{\ell}\right|^{2}\right\}+\omega\right) \\
& \quad \times \mathrm{E}\left\{\frac{1}{\kappa p_{\mathrm{a}} \beta_{\mathrm{au}, k}\left(K_{\mathrm{ul}}+N-1\right)\left\|\mathbf{g}_{\mathrm{au}, k}\right\|^{2}}\right\} \\
& \stackrel{(a)}{=} \frac{\kappa p_{\mathrm{a}}\left(K_{\mathrm{ul}}+N-1\right) \sum_{\ell \neq k}^{K_{\mathrm{ul}}} \beta_{\mathrm{au}, \ell}^{2}+\omega}{\kappa p_{\mathrm{a}} \beta_{\mathrm{au}, k}\left(K_{\mathrm{ul}}+N-1\right)} \mathrm{E}\left\{\frac{1}{\mathbf{g}_{\mathrm{au}, k}^{\dagger} \mathbf{g}_{\mathrm{au}, k}}\right\} \\
& \stackrel{(b)}{=} \frac{\kappa p_{\mathrm{a}}\left(K_{\mathrm{ul}}+N-1\right) \sum_{\ell \neq k}^{K_{\mathrm{ul}}} \beta_{\mathrm{au}, \ell}^{2}+\omega}{\kappa p_{\mathrm{a}} \beta_{\mathrm{au}, k}^{2}\left(K_{\mathrm{ul}}+N-1\right)(N-1)},
\end{aligned}
$$

where (a) follows from the fact that $\hat{g}_{\ell} \sim \mathcal{C N}\left(0, \beta_{\mathrm{au}, \ell}\right)$ and (b) follows by using the identity [41, Lemma 2.10]

$$
\mathrm{E}\left\{\operatorname{tr}\left(\mathbf{W}^{-1}\right)\right\}=\frac{m}{n-m},
$$

where $\mathbf{W} \sim \mathcal{W}_{m}\left(n, \mathbf{I}_{n}\right)$ is an $m \times m$ central complex Wishart matrix with $n(n>m)$ degrees-of-freedom. Finally, by substituting (52) into (51) we arrive at the desired result.

\section{APPENDIX C}

\section{PROOF OF COROLlARY 1}

With the genie aided receiver at the HAP, we have $\hat{\mathrm{g}}_{\mathrm{ad}, k}=$ $\mathbf{g}_{\mathrm{ad}, k}$, i.e., $\boldsymbol{\varepsilon}_{\mathrm{ad}, k}=0$. Hence, the $k$-th stream of $\mathbf{r}_{\mathrm{a}}[i]$ in (7) can be expressed as

$$
\begin{aligned}
r_{\mathrm{a}, k}[i]= & \mathbf{w}_{r, k}^{\dagger} \mathbf{g}_{\mathrm{ua}, k} x_{\mathrm{u}, k}[i]+\sum_{\ell \neq k}^{K_{\mathrm{ul}}} \mathbf{w}_{r, k}^{\dagger} \mathbf{g}_{\mathrm{ua}, \ell} x_{\mathbf{u}, \ell}[i] \\
& +\sum_{\ell=1}^{K_{\mathrm{dll}}} \mathbf{w}_{r, k}^{\dagger} \mathbf{H}_{\mathrm{Sl}}^{T} \mathbf{w}_{t, \ell} x_{\mathrm{d}, \ell}[i]+\mathbf{w}_{r, k}^{\dagger} \mathbf{n}_{\mathrm{a}}[i] .
\end{aligned}
$$

Moreover, the SI term $\mathbf{w}_{r, k}^{\dagger} \mathbf{H}_{\mathrm{SI}} \mathbf{w}_{t, \ell} x_{\mathrm{d}, \ell}[i]$ is known at the HAP and known interference cancellation technique can be applied to cancel this term. However, due to hardware impairments at the full-duplex transceiver, the SI term cannot be perfectly removed. According to [5], the signal after performing known interference cancellation can be modeled by

$$
\begin{aligned}
r_{\mathrm{a}, k}[i]= & \mathbf{w}_{r, k}^{\dagger} \mathbf{g}_{\mathrm{ua}, k} x_{\mathrm{u}, k}[i]+\sum_{\ell \neq k}^{K_{\mathrm{ul}}} \mathbf{w}_{r, k}^{\dagger} \mathbf{g}_{\mathrm{ua}, \ell} x_{\mathbf{u}, \ell}[i] \\
& +\sum_{\ell=1}^{K_{\mathrm{dl}}} \mathbf{w}_{r, k}^{\dagger} \tilde{\mathbf{H}}_{\mathrm{SI}}^{T} \mathbf{w}_{t, \ell} x_{\mathrm{d}, \ell}[i]+\mathbf{w}_{r, k}^{\dagger} \mathbf{n}_{\mathrm{a}}[i],
\end{aligned}
$$

where $\tilde{\mathbf{H}}_{\mathrm{SI}} \sim \mathcal{C N}\left(0, k_{1} \sigma_{\mathrm{SI}}^{2}\right)$. Accordingly, by substituting $\hat{\mathbf{w}}_{t, k}=\mathbf{w}_{t, k}^{\mathrm{MRT}}$ into (9), the uplink spectral efficiency can be expressed as (56) at the top of the page. Moreover, we get

$\mathrm{E}_{\tilde{\mathbf{H}}_{\mathrm{SI}}}\left\{\left|\mathbf{w}_{r, k}^{\dagger} \tilde{\mathbf{H}}_{\mathrm{SI}}^{T} \mathbf{w}_{t, \ell}\right|^{2} \mid \mathbf{w}_{r, k}, \mathbf{w}_{t, \ell}\right\}=k_{1} \sigma_{\mathrm{SI}}^{2}\left\|\mathbf{w}_{r, k}\right\|^{2}\left\|\mathbf{w}_{t, \ell}\right\|^{2}$.

To this end, following similar steps to those of Proposition 2, the desired result in (18) is obtained.

\section{APPENDIX D \\ PROOF OF PROPOSITION 3}

By substituting $\hat{\mathbf{w}}_{t, k}^{\mathrm{MRT}}$ into (12), and recalling that $p_{\mathrm{u}, \ell} \approx$ $\kappa p_{\mathrm{a}} \beta_{\mathrm{au}, \ell}\left(K_{\mathrm{ul}}+N-1\right)$ for $N \rightarrow \infty$, the downlink spectral efficiency can be expressed as (58) at the top of the next page, where $\breve{g}_{\ell}=\frac{\hat{\mathrm{g}}_{\mathrm{ad}, k} \hat{\mathrm{g}}_{\mathrm{ad}, \ell}^{\dagger}}{\left\|\hat{\mathrm{g}}_{\mathrm{ad}, k}\right\|}$ and $\Gamma=\kappa\left(K_{\mathrm{ul}}+N-\right.$ 1) $\sum_{l=1}^{K_{\mathrm{ul}}} \beta_{\mathrm{ud}, k \ell} \beta_{\mathrm{au}, \ell}+\frac{1}{\rho_{\mathrm{a}}}$. Now, by using the approximation $\mathrm{E}\left\{\log _{2}\left(1+\frac{X}{Y}\right)\right\} \approx \log _{2}\left(1+\frac{\mathrm{E}\{X\}}{\mathrm{E}\{Y\}}\right)$ [34, Lemma 1], we arrive at (60) at the top of the page.

Note that conditioned on $\mathbf{g}_{\mathrm{ad}, k}, \breve{g}_{\ell}$ is a zero-mean Gaussian RV with unit variance which does not depend on $\mathbf{g}_{\mathrm{ad}, k}$. 


$$
R_{\mathrm{ul}, \mathrm{k}}^{\mathrm{MR}}=(1-\alpha) \sum_{k=1}^{K_{\mathrm{ul}}} \mathrm{E}\left\{\log _{2}\left(1+\frac{\mathrm{p}_{\mathrm{u}, \mathrm{k}}\left|\mathbf{w}_{\mathrm{r}, \mathrm{k}}^{\dagger} \mathbf{g}_{\mathrm{ua}, \mathrm{k}}\right|^{2}}{\sum_{\ell \neq \mathrm{k}}^{\mathrm{K}_{\mathrm{ul}}} \mathrm{p}_{\mathrm{u}, \ell}\left|\mathbf{w}_{\mathrm{r}, \mathrm{k}}^{\dagger} \mathbf{g}_{\mathrm{ua}, \ell}\right|^{2}+\mathrm{p}_{\mathrm{a}} \sum_{\ell=1}^{\mathrm{K}_{\mathrm{dl}}} \mathrm{E}_{\tilde{\mathbf{H}}_{\mathrm{sl}}}\left\{\left|\mathbf{w}_{\mathrm{r}, \mathrm{k}}^{\dagger} \tilde{\mathbf{H}}_{\mathrm{SI}}^{\mathrm{T}} \mathbf{w}_{\mathrm{t}, \ell}\right|^{2} \mid \mathbf{w}_{\mathrm{r}, \mathrm{k}}, \mathbf{w}_{\mathrm{t}, \ell}\right\}+\sigma_{\mathrm{n}}^{2}}\right)\right\}
$$

$$
\begin{aligned}
& R_{\mathrm{dl}, \mathrm{k}}^{\mathrm{MR}}=(1-\alpha) \mathrm{E}\left\{\log _{2}\left(1+\frac{\left\|\hat{\mathbf{g}}_{\mathrm{ad}, k}\right\|^{2}}{\sum_{\ell \neq k}^{K_{\mathrm{d} \|}}\left|\breve{g}_{\ell}\right|^{2}+\left(\beta_{\mathrm{ad}, k}-\sigma_{\mathrm{ad}, k}^{2}\right) \sum_{\ell=1}^{K_{\mathrm{dl}}} \frac{\left\|\hat{\mathrm{g}}_{\mathrm{a} d}, \ell\right\|^{2}}{\left\|\hat{\mathrm{g}}_{\mathrm{ad}, k}\right\|^{2}}+\frac{\Gamma}{\alpha_{\mathrm{MRT}}^{2}\left\|\hat{\mathrm{g}}_{\mathrm{ad}, k}\right\|^{2}}}\right)\right\},
\end{aligned}
$$

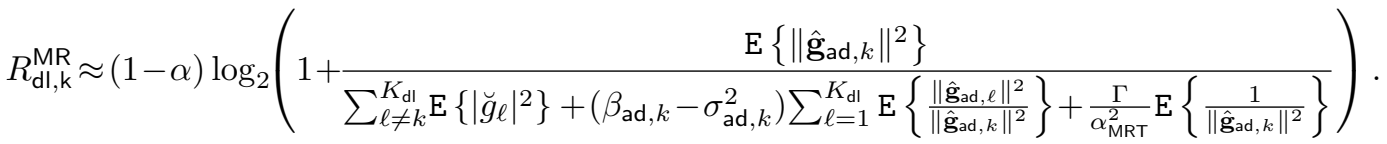

Hence, $\breve{g}_{\ell} \sim \mathcal{C N}\left(0, \sigma_{\text {ad }, \ell}^{2}\right)$, which results in $\mathrm{E}\left\{\left|\breve{g}_{\ell}\right|^{2}\right\}=$ $\sigma_{\mathrm{ad}, \ell}^{2}$. Moreover, from (53), we have $\mathrm{E}\left\{\frac{1}{\left\|\hat{\mathrm{g}}_{\mathrm{ad}, k}\right\|^{2}}\right\}=$ $\frac{1}{(M-1) \sigma_{\mathrm{ad}, k}^{2}}$. Furthermore, since $\hat{\mathrm{g}}_{\mathrm{ad}, \ell}$ and $\hat{\mathrm{g}}_{\mathrm{ad}, k}$ are independent $\mathrm{RVs}$, we have

$$
\begin{aligned}
\mathrm{E}\left\{\frac{\left\|\hat{\mathbf{g}}_{\mathrm{ad}, \ell}\right\|^{2}}{\left\|\hat{\mathrm{g}}_{\mathrm{ad}, k}\right\|^{2}}\right\} & =\mathrm{E}\left\{\left\|\hat{\mathbf{g}}_{\mathrm{ad}, \ell}\right\|^{2}\right\} \mathrm{E}\left\{\frac{1}{\left\|\hat{\mathbf{g}}_{\mathrm{ad}, k}\right\|^{2}}\right\} \\
& =\frac{M}{M-1} \frac{\sigma_{\mathrm{ad}, \ell}^{2}}{\sigma_{\mathrm{ad}, k}^{2}}
\end{aligned}
$$

where we used the identity (53). To this end, by substituting the corresponding expectations into (60), the desired result in (19) is obtained.

\section{APPENDIX E}

\section{PROOF OF PROPOSITION 4}

By substituting $\mathbf{W}_{r}^{\mathrm{ZF}}$ and $p_{\mathrm{u}, k}$ into (9), the uplink achievable rate from the $k$-th UE can be expressed as (61) at the top of the next page.

When $N$ grows large, according to (47), we have $\sum_{\ell \neq k}^{K_{\mathrm{ul}}} \frac{\mid \mathbf{g}_{\mathrm{au}, k} \mathbf{g}_{\mathrm{au}, \ell^{\dagger}}^{\dagger}}{\left\|\mathbf{g}_{\mathrm{au}, \ell}\right\|^{2}} \stackrel{N \rightarrow \infty}{\longrightarrow} \beta_{\mathrm{au}, k}\left(K_{\mathrm{ul}}-1\right)$ and $\frac{1}{N}\left\|\mathbf{g}_{\mathrm{au}, k}\right\| \stackrel{N \rightarrow \infty}{\longrightarrow} \beta_{\mathrm{au}, k}$. Therefore, the achievable rate in (61) can be written as (62) at the top of the page.

Let us denote $Z_{k}=\mathrm{E}_{\tilde{\mathcal{E}}_{\mathrm{SI}}}\left\{\left|\mathbf{w}_{r, k}^{\dagger} \tilde{\mathcal{E}}_{\mathrm{SI}}^{T} \hat{\mathbf{w}}_{t, \ell}\right|^{2} \mid \mathbf{w}_{r, k}, \hat{\mathbf{w}}_{t, \ell}\right\}$. Conditioned on $\mathbf{w}_{r, k}, \mathbf{w}_{r, k}^{\dagger} \tilde{\mathcal{E}}_{\mathrm{SI}}^{T}$ is a Gaussian random vector with zero mean and covariance matrix $\left\|\mathbf{w}_{r, k}\right\|^{2}\left(k_{1} \sigma_{\mathrm{SI}}^{2}+\right.$ $\left.\frac{1}{1+\tau p_{\mathrm{a}} \sigma_{\mathrm{Sl}}^{2}}\right) \mathbf{I}_{M}$. Since the covariance matrix does not depend on $\mathbf{w}_{r, k}, \mathbf{w}_{r, k}^{\dagger} \tilde{\mathcal{E}}_{\mathrm{SI}}^{T}$ is a Gaussian distributed vector and independent of $\mathbf{w}_{r, k}$. Therefore, $Z_{k}=\mathrm{E}_{\tilde{\mathcal{E}}_{\mathrm{SI}}}\left\{\left|\overline{\mathcal{E}}_{\mathrm{SI}} \hat{\mathbf{w}}_{t, \ell}\right|^{2} \mid \hat{\mathbf{w}}_{t, \ell}\right\}$ where $\overline{\mathcal{E}}_{\mathrm{SI}}=\mathbf{w}_{r, k}^{\dagger} \tilde{\mathcal{E}}_{\mathrm{SI}}^{T}$. Accordingly, we can obtain

$$
\begin{aligned}
& Z_{k}=\mathrm{E}_{\overline{\mathcal{E}}_{\mathrm{SI}}}\left\{\hat{\mathbf{w}}_{t, \ell}^{\dagger} \overline{\mathcal{E}}_{\mathrm{SI}} \overline{\mathcal{E}}_{\mathrm{SI} \mid} \hat{\mathbf{w}}_{t, \ell} \mid \hat{\mathbf{w}}_{t, \ell}\right\} \\
& =\left(k_{1} \sigma_{\mathrm{SI}}^{2}+\frac{1}{1+\tau p_{\mathrm{a}} \sigma_{\mathrm{SI}}^{2}}\right)\left\|\mathbf{w}_{r, k}\right\|^{2}\left\|\hat{\mathbf{w}}_{t, \ell}\right\|^{2} .
\end{aligned}
$$

To this end, by substituting (63) into (62) and then by using the convexity of $\log _{2}\left(1+\frac{1}{x}\right)$, and employing Jensen's inequality, the lower bound on the uplink achievable rate from the $k$-th UE is achieved as (64) at the top of the next page. To this end, since $\mathbf{w}_{r, k}$ and $\hat{\mathbf{w}}_{t, \ell}$ are independent, $\mathrm{E}\left\{\left\|\mathbf{w}_{r, k}\right\|^{2}\left\|\hat{\mathbf{w}}_{t, \ell}\right\|^{2}\right\}=\mathrm{E}\left\{\left\|\mathbf{w}_{r, k}\right\|^{2}\right\} \mathrm{E}\left\{\left\|\hat{\mathbf{w}}_{t, \ell}\right\|^{2}\right\}$. Now we proceed to derive $\mathrm{E}\left\{\left\|\mathbf{w}_{r, k}\right\|^{2}\right\}$ and $\mathrm{E}\left\{\left\|\hat{\mathbf{w}}_{t, \ell}\right\|^{2}\right\}$, respectively. From (21a), we have

$$
\begin{aligned}
\mathrm{E}\left\{\left\|\mathbf{w}_{r, k}\right\|^{2}\right\} & =\alpha_{\mathrm{RZF}}^{2} \mathrm{E}\left\{\left[\left(\mathbf{G}_{\mathrm{au}}^{\dagger} \mathbf{G}_{\mathrm{au}}\right)^{-1}\right]_{k k}\right\} \\
& =\frac{\alpha_{\mathrm{RZF}}^{2}}{K_{\mathrm{ul}} \beta_{\mathrm{au}, k}} \mathrm{E}\left\{\operatorname{tr}\left[\left(\mathbf{H}_{\mathrm{au}}^{\dagger} \mathbf{H}_{\mathrm{au}}\right)^{-1}\right]\right\} \\
& =\frac{\alpha_{\mathrm{RZF}}^{2}}{\beta_{\mathrm{au}, k}} \frac{1}{N-K_{\mathrm{ul}}}, \quad \text { for } \quad N \geq K_{\mathrm{ul}}
\end{aligned}
$$

where the last equality is obtained by using (53). Moreover, $\mathrm{E}\left\{\left\|\hat{\mathbf{w}}_{t, \ell}\right\|^{2}\right\}$ can be similarly obtained as

$$
\mathrm{E}\left\{\left\|\hat{\mathbf{w}}_{t, \ell}\right\|^{2}\right\}=\frac{\alpha_{\mathrm{TZF}}^{2}}{\sigma_{\mathrm{ad}, \ell}^{2}} \frac{1}{M-K_{\mathrm{dl}}}, \quad \text { for } \quad M \geq K_{\mathrm{dl}} .
$$

Finally, by substituting (65) and (66) into (64), following by some algebraic manipulation, the desired result in (23) is obtained.

\section{REFERENCES}

[1] M. Mohammadi, B. K. Chalise, H. A. Suraweera, and Z. Ding, "Wireless information and power transfer in full-duplex systems with massive antenna arrays," in Proc. IEEE Intl. Conf. Commun. (ICC'17), Paris, France, May 2017, pp. 1-6.

[2] E. G. Larsson, O. Edfors, F. Tufvesson, and T. L. Marzetta, "Massive MIMO for next generation wireless systems," IEEE Commun. Mag., vol. 52, no. 2, pp. 186-195, Feb. 2014.

[3] X. Lu, P. Wang, D. Niyato, D. I. Kim, and Z. Han, "Wireless networks with RF energy harvesting: A contemporary survey," IEEE Commun. Surveys Tuts, vol. 17, pp. 757-789, Secondquarter 2015.

[4] A. Sabharwal, P. Schniter, D. Guo, D. W. Bliss, S. Rangarajan, and R. Wichman, "In-band full-duplex wireless: Challenges and opportunities," IEEE J. Sel. Areas Commun., vol. 32, pp. 1637-1652, Sep. 2014.

[5] T. Riihonen, S. Werner, and R. Wichman, "Mitigation of loopback self-interference in full-duplex MIMO relays," IEEE Trans. Signal Process., vol. 59, pp. 5983-5993, Dec. 2011.

[6] M. Duarte, C. Dick, and A. Sabharwal, "Experiment-driven characterization of full-duplex wireless systems," IEEE Trans. Wireless Commun., vol. 11, pp. 4296-4307, Dec. 2012.

[7] M. Mohammadi, H. A. Suraweera, Y. Cao, I. Krikidis, and C. Tellambura, "Full-duplex radio for uplink/downlink wireless access with spatially random nodes," IEEE Trans. Commun., vol. 63, no. 12, pp. 5250-5266, Dec. 2015.

[8] E. Everett, A. Sahai, and A. Sabharwal, "Passive self-interference suppression for full-duplex infrastructure nodes," IEEE Trans. Wireless Commun., vol. 13, no. 2, pp. 680-694, Feb. 2014.

[9] H. Q. Ngo, H. A. Suraweera, M. Matthaiou, and E. G. Larsson, "Multipair full-duplex relaying with massive arrays and linear processing," IEEE J. Sel. Areas Commun., vol. 32, pp. 1721-1737, June 2014.

[10] J. Bai and A. Sabharwal, "Asymptotic analysis of MIMO multi-cell full-duplex networks," IEEE Trans. Wireless Commun., vol. 16, pp. 2168-2180, Apr. 2017. 


$$
R_{\mathrm{ul}, \mathrm{k}}^{\mathrm{ZF}} \approx(1-\alpha) \mathrm{E}\left\{\log _{2}\left(1+\frac{\kappa p_{\mathrm{a}} \alpha_{\mathrm{RZF}}^{2}\left(\sum_{\ell=1, \ell \neq k}^{K_{\mathrm{ul}}}\left|\frac{\mathbf{g}_{\mathrm{au}, k} \mathbf{g}_{\mathrm{au}, \ell}^{\dagger}}{\left\|\mathbf{g}_{\mathrm{au}, \ell}\right\|}\right|^{2}+\left\|\mathbf{g}_{\mathrm{au}, k}\right\|^{2}\right)}{p_{\mathrm{a}} \sum_{\ell=1}^{K_{\mathrm{dll}}} \mathrm{E}_{\tilde{\mathcal{E}}_{\mathrm{sl}}}\left\{\left|\mathbf{w}_{r, k}^{\dagger} \tilde{\mathcal{E}}_{\mathrm{SI}}^{T} \hat{\mathbf{w}}_{t, \ell}\right|^{2} \mid \mathbf{w}_{r, k}, \hat{\mathbf{w}}_{t, \ell}\right\}+\sigma_{n}^{2}\left\|\mathbf{w}_{r, k}\right\|^{2}}\right)\right\} .
$$

$$
R_{\mathrm{ul}, \mathrm{k}}^{\mathrm{ZF}} \approx(1-\alpha) \mathrm{E}\left\{\log _{2}\left(1+\frac{\kappa p_{\mathrm{a}} \alpha_{\mathrm{RZF}}^{2} \beta_{\mathrm{au}, k}\left(K_{\mathrm{ul}}+N-1\right)}{p_{\mathrm{a}} \sum_{\ell=1}^{K_{\mathrm{dl}}} \mathrm{E}_{\tilde{\mathcal{E}}_{\mathrm{Sl}}}\left\{\left|\mathbf{w}_{r, k}^{\dagger} \tilde{\mathcal{E}}_{\mathrm{SI}}^{T} \hat{\mathbf{w}}_{t, \ell}\right|^{2} \mid \mathbf{w}_{r, k}, \hat{\mathbf{w}}_{t, \ell}\right\}+\sigma_{n}^{2}\left\|\mathbf{w}_{r, k}\right\|^{2}}\right)\right\} .
$$

$$
R_{\mathrm{ul}, \mathrm{k}}^{\mathrm{ZF}} \geq \tilde{R}_{\mathrm{ul}, \mathrm{k}}^{\mathrm{ZF}}=(1-\alpha) \log _{2}\left(1+\left(\frac{\mathrm{E}\left\{p_{\mathrm{a}}\left(k_{1} \sigma_{\mathrm{SI}}^{2}+\frac{1}{1+\tau p_{\mathrm{a}} \sigma_{\mathrm{S}}^{2}}\right)\left\|\mathbf{w}_{r, k}\right\|^{2} \sum_{\ell=1}^{K_{\mathrm{dl}}}\left\|\hat{\mathbf{w}}_{t, \ell}\right\|^{2}+\sigma_{n}^{2}\left\|\mathbf{w}_{r, k}\right\|^{2}\right\}}{\kappa p_{\mathrm{a}} \alpha_{\mathrm{RZF}}^{2} \beta_{\mathrm{au}, k}\left(K_{\mathrm{ul}}+N-1\right)}\right)^{-1}\right) .
$$

[11] C. Psomas, M. Mohammadi, I. Krikidis, and H. A. Suraweera, "Impact of directionality on interference mitigation in full-duplex cellular networks," IEEE Trans. Wireless Commun., vol. 16, no. 1, pp. 487502, Jan 2017.

[12] A. C. Cirik, M. J. Rahman, and L. Lampe, "Robust fairness transceiver design for a full-duplex MIMO multi-cell system," IEEE Trans. Commun., vol. 66, no. 3, pp. 1027-1041, Mar. 2018.

[13] I. Randrianantenaina, H. ElSawy, H. Dahrouj, M. Kaneko, and M. Alouini, "Uplink power control and ergodic rate characterization in FD cellular networks: A stochastic geometry approach," IEEE Trans. Wireless Commun., vol. 18, pp. 2093-2110, Apr. 2019.

[14] G. Yang, C. K. Ho, R. Zhang, and Y. L. Guan, "Throughput optimization for massive MIMO systems powered by wireless energy transfer," IEEE J. Sel. Areas Commun., vol. 33, pp. 1640-1650, Aug. 2015.

[15] L. Zhao, X. Wang, and K. Zheng, "Downlink hybrid information and energy transfer with massive MIMO," IEEE Trans. Wireless Commun., vol. 15, pp. 1309-1322, Feb. 2016.

[16] S. Kashyap, E. Björnson, and E. G. Larsson, "On the feasibility of wireless energy transfer using massive antenna arrays," IEEE Trans. Wireless Commun., vol. 15, no. 5, pp. 3466-3480, May 2016.

[17] X. Wang and C. Zhai, "Simultaneous wireless information and power transfer for multi-user massive antenna-array systems," IEEE Trans. Commun., vol. 65, pp. 4039 - 4048, Sept. 2017.

[18] L. Zhao, K. Zheng, H. Yang, and W. Xiang, "Beamformer design and utility optimization for hybrid information and energy transfer with massive MIMO," IEEE Syst. J., vol. 12, pp. 1981 - 1992, June 2018.

[19] Z. Chang, Z. Wang, X. Guo, Z. Han, and T. Ristaniemi, "Energyefficient resource allocation for wireless powered massive MIMO system with imperfect CSI," IEEE Trans. Green Commun. and Networking, vol. 1, no. 2, pp. 121-130, June 2017.

[20] L. Zhao and X. Wang, "Massive MIMO downlink for wireless information and energy transfer with energy harvesting receivers," IEEE Trans. Commun., vol. 67, pp. 3309-3322, May 2019.

[21] V. D. Nguyen, T. Q. Duong, H. D. Tuan, O. S. Shin, and H. V. Poor, "Spectral and energy efficiencies in full-duplex wireless information and power transfer," IEEE Trans. Commun., vol. 65, no. 5, pp. 22202233, May 2017.

[22] K. Xu, Z. Shen, Y. Wang, X. Xia, and D. Zhang, "Hybrid timeswitching and power splitting SWIPT for full-duplex massive MIMO systems: A beam-domain approach," IEEE Trans. Veh. Technol., vol. 67, no. 8, pp. 7257-7274, Aug 2018.

[23] D. S. Gurjar, H. H. Nguyen, and H. D. Tuan, "Wireless information and power transfer for IoT applications in overlay cognitive radio networks," IEEE Internet of Things Journal, vol. 6, no. 2, pp. 3257 3270, Apr. 2019.

[24] X. Wang, A. Ashikhmin, and X. Wang, "Wirelessly powered cell-free IoT: Analysis and optimization," IEEE Internet of Things Journal, pp. $1-1,2020$.

[25] H. Huh, G. Caire, H. C. Papadopoulos, and S. A. Ramprashad, "Achieving "massive MIMO" spectral efficiency with a not-so-large number of antennas," IEEE Trans. Wireless Commun., vol. 11, no. 9, pp. 3226-3239, Sept. 2012.

[26] S. M. Kay, Fundamentals of Statistical Signal Processing: Estimation Theory. Englewood Cliffs, NJ: Prentice Hall, 1993.

[27] R. Zhang and C. K. Ho, "MIMO broadcasting for simultaneous wireless information and power transfer," IEEE Trans. Wireless Commun, vol. 12, pp. 1989-2001, May 2013.

[28] A. A. Nasir, X. Zhou, S. Durrani, and R. A. Kennedy, "Relaying protocols for wireless energy harvesting and information processing," IEEE Trans. Wireless Commun., vol. 12, pp. 3622-3636, July 2013.

[29] H. Q. Ngo, E. G. Larsson, and T. L. Marzetta, "Energy and spectral efficiency of very large multiuser MIMO systems," IEEE Trans. Commun., vol. 61, pp. 1436-1449, Apr. 2013.

[30] J. Hoydis, S. ten Brink, and M. Debbah, "Massive MIMO in the UL/DL of cellular networks: How many antennas do we need?" IEEE J. Sel. Areas Commun., vol. 31, no. 2, pp. 160-171, Feb. 2013.

[31] C. Kong, C. Zhong, A. K. Papazafeiropoulos, M. Matthaiou, and Z. Zhang, "Sum-rate and power scaling of massive MIMO systems with channel aging," IEEE Trans. Commun., vol. 63, no. 12, pp. 48794893, Dec. 2015.

[32] L. Van der Perre, L. Liu, and E. G. Larsson, "Efficient DSP and circuit architectures for massive MIMO: State of the art and future directions," IEEE Trans. Signal Process., vol. 66, no. 18, pp. 47174736, Sept. 2018.

[33] H. Yang and T. L. Marzetta, "Performance of conjugate and zeroforcing beamforming in large-scale antenna systems," IEEE J. Sel. Areas Commun., vol. 31, pp. 172-179, Feb. 2013.

[34] Q. Zhang, S. Jin, K. Wong, H. Zhu, and M. Matthaiou, "Power scaling of uplink massive MIMO systems with arbitrary-rank channel means," J. Sel. Topics Signal Processing, vol. 8, no. 5, pp. 966-981, Oct. 2014.

[35] H. Prabhu, J. Rodrigues, O. Edfors, and F. Rusek, "Approximative matrix inverse computations for very-large MIMO and applications to linear pre-coding systems," in Proc. IEEE Wireless Commun. and Networking Conf. (WCNC'13), Shanghai, China, Apr. 2013, pp. 27102715.

[36] H. Y. T. L. Marzetta, E. G. Larsson and H. Q. Ngo, Fundamentals of massive MIMO. Cambridge University Press, 2016.

[37] G. A. F. Seber, A Matrix Handbook for Statisticians. Hoboken, NJ: John Wiley \& Sons, Inc., 2008.

[38] B. K. Chalise and L. Vandendorpe, "MIMO relay design for multipoint-to-multipoint communications with imperfect channel state information," IEEE Trans. Signal Process., vol. 57, pp. 2785-2796, July 2009.

[39] H. Q. Ngo, A. Ashikhmin, H. Yang, E. G. Larsson, and T. L. Marzetta, "Cell-free massive MIMO versus small cells," IEEE Trans. Wireless Commun., vol. 16, pp. 1834-1850, Mar. 2017.

[40] K. A. Hamdi, "A useful lemma for capacity analysis of fading interference channels," IEEE Trans. Commun., vol. 58, pp. 411-416, Feb. 2010.

[41] A. Tulino and S. Verdú, Random Matrix Theory and Wireless Communications. Delft, Netherlands: Now Publishers Inc., 2004. 